\title{
RISE: An Incremental Trust-Region Method for Robust Online Sparse Least-Squares Estimation
}

\author{
David M. Rosen, Student Member, IEEE, Michael Kaess, Member, IEEE, \\ and John J. Leonard, Fellow, IEEE
}

\begin{abstract}
Many point estimation problems in robotics, computer vision and machine learning can be formulated as instances of the general problem of minimizing a sparse nonlinear sum-ofsquares objective function. For inference problems of this type, each input datum gives rise to a summand in the objective function, and therefore performing online inference corresponds to solving a sequence of sparse nonlinear least-squares minimization problems in which additional summands are added to the objective function over time. In this paper we present Robust Incremental least-Squares Estimation (RISE), an incrementalized version of the Powell's Dog-Leg numerical optimization method suitable for use in online sequential sparse least-squares minimization. As a trust-region method, RISE is naturally robust to objective function nonlinearity and numerical ill-conditioning, and is provably globally convergent for a broad class of inferential cost functions (twice-continuously differentiable functions with bounded sublevel sets). Consequently, RISE maintains the speed of current state-of-the-art online sparse least-squares methods while providing superior reliability.
\end{abstract}

Index Terms-Sparse least-squares minimization, online estimation, SLAM, computer vision, machine learning

\section{INTRODUCTION}

$\mathbf{M}$ ANY point estimation problems in robotics, computer vision and machine learning can be formulated as instances of the general problem of minimizing a sparse nonlinear sum-of-squares objective function; for example, the archetypal problems of full (smoothing) simultaneous localization and mapping (SLAM) [1] (in robotics), bundle adjustment (BA) [2], [3] (in computer vision), and sparse (kernel) regularized least-squares classification and regression [4], [5] (in machine learning) all belong to this class. For inference problems of this type, each input datum gives rise to a summand in the objective function, and therefore performing online inference (in which the data is collected sequentially and the estimate updated after the incorporation of each new datum) corresponds to solving a sequence of sparse leastsquares minimization problems in which additional summands are added to the objective function over time.

In practice, these online inference problems are often solved by computing each estimate in the sequence as the solu-

Received Aug. 5, 2013; revised Jan. 9, 2014; accepted Mar. 31, 2014.

D.M. Rosen and J.J. Leonard are with the Computer Science and Artificial Intelligence Laboratory of the Massachusetts Institute of Technology, Cambridge, MA 02139, USA. Email: $\{$ dmrosen |jleonard $\}$ amit. edu.

M. Kaess is with the Robotics Institute at Carnegie Mellon University, Pittsburgh, PA 15213, USA. Email: kaess@ cmu .edu.

This work was partially supported by Office of Naval Research (ONR) grants N00014-12-1-0093, N00014-11-1-0688, N00014-06-1-0043 and N00014-10-1-0936, and by Air Force Research Laboratory (AFRL) contract FA8650-11-C-7137.

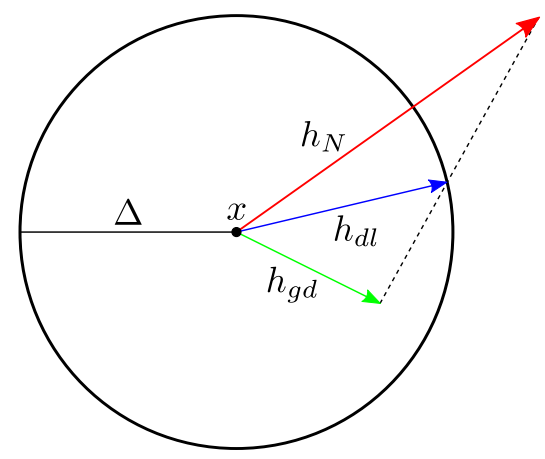

Fig. 1. The Powell's Dog-Leg update step $h_{d l}$ is obtained by interpolating the (possibly approximate) Newton step $h_{N}$ and the gradient descent step $h_{g d}$ using a trust-region of radius $\Delta$ centered on the current iterate $x$. By adapting $\Delta$ online in response to the observed performance of the Newton steps near $x$, the algorithm is able to combine the rapid end-stage convergence speed of Newton-type methods with the reliability of gradient descent.

tion of an independent minimization problem using standard sparse least-squares techniques (most commonly LevenbergMarquardt [6]-[8]). While this approach is general and produces good results, it is computationally expensive, and does not exploit the sequential structure of the underlying inference problem; this limits its utility in real-time online applications, where speed is crucial.

More sophisticated solutions achieve faster computation by directly exploiting the sequentiality of the online inference problem. The canonical example is online gradient descent, which is attractive for its robustness, simplicity, and low memory and per-iteration computational costs, but its firstorder rate can lead to painfully slow convergence [8]. Alternatively, Kaess et al. have developed incremental smoothing and mapping (iSAM), [9], [10], which exploits recent algorithmic advances in sparse numerical linear algebra to implement an efficient incrementalized version of the GaussNewton method [8] for use in online sparse least-squares minimization. This incremental approach, together with the Gauss-Newton method's superlinear convergence rate, enables iSAM to achieve computational speeds unmatched by iterated batch techniques. However, the Gauss-Newton method can exhibit poor (even globally divergent) behavior when applied to objective functions with significant nonlinearity [11], which restricts the class of problems to which iSAM can be reliably applied. To date, the development of a fully incremental online sparse least-squares solver that combines the robustness of gradient descent with the superlinear convergence rate of Newton-type methods has remained an outstanding problem. 
To that end, in this paper we present Robust Incremental least-Squares Estimation (RISE), an incrementalized version of the Powell's Dog-Leg numerical optimization algorithm [8], [12] suitable for use in online sequential sparse least-squares minimization. As a trust-region method (Fig. 1), Powell's Dog-Leg is naturally robust to objective function nonlinearity and numerical ill-conditioning, and enjoys excellent global convergence properties [13]-[15]; furthermore, it is known to perform significantly faster than Levenberg-Marquardt in batch sparse least-squares minimization while obtaining solutions of comparable quality [16]. By exploiting iSAM's pre-existing functionality to incrementalize the computation of the dogleg step, RISE maintains the speed of current state-of-the-art online sparse least-squares solvers while providing superior robustness to objective function nonlinearity and numerical ill-conditioning.

The rest of this paper is organized as follows. In the next section we formulate the sequential sparse least-squares minimization problem and discuss its connections to online inference. In Section III we review the class of Newton-type optimization methods, focusing in particular on the Gauss-Newton method and its incrementalization to produce iSAM. Section IV introduces the general class of trust-region methods, paying particular attention to Powell's Dog-Leg. Here we derive the indefinite Gauss-Newton-Powell's Dog-Leg (IGN-PDL) algorithm (an extension of Powell's Dog-Leg with GaussNewton steps to the case of indefinite Jacobians), analyze its robustness with respect to objective function nonlinearity and numerical ill-conditioning, and establish sufficient conditions for its global convergence (Theorem 3). We then derive the RISE and RISE2 algorithms in Section V by incrementalizing IGN-PDL with the aid of iSAM. We contextualize RISE with a discussion of related work in Section VI, and evaluate its performance in Section VII on standard 6DOF pose-graph SLAM benchmarks and on a real-world visual mapping task using a calibrated monocular camera. Finally, Section VIII concludes with a summary of this paper's contributions and a discussion of future research directions.

\section{PROBLEM FORMULATION}

We are interested in the general problem of obtaining a point estimate $x^{*} \in \mathbb{R}^{n}$ of some quantity of interest $X$ as the solution of a sparse nonlinear least-squares problem

$$
\begin{gathered}
\min _{x \in \mathbb{R}^{n}} S(x) \\
S(x)=\sum_{i=1}^{m} r_{i}(x)^{2}=\|r(x)\|^{2}
\end{gathered}
$$

for $r: \mathbb{R}^{n} \rightarrow \mathbb{R}^{m}$ with $m \geq n$. Problems of this form frequently arise in probabilistic inference in the context of maximum likelihood (ML) or maximum a posteriori (MAP) parameter estimation; indeed, performing ML or MAP estimation over any probability distribution $p: \mathbb{R}^{n} \rightarrow \mathbb{R}^{+}$whose factor graph representation $\mathcal{G}=(\mathcal{F}, \mathcal{X}, \mathcal{E})$ [17] is sparse and whose factors are positive and bounded is equivalent to solving a sparse least-squares problem of the form (1) in which each summand $r_{i}$ corresponds to a factor $p_{i}$ of $p$ [18]. Given the

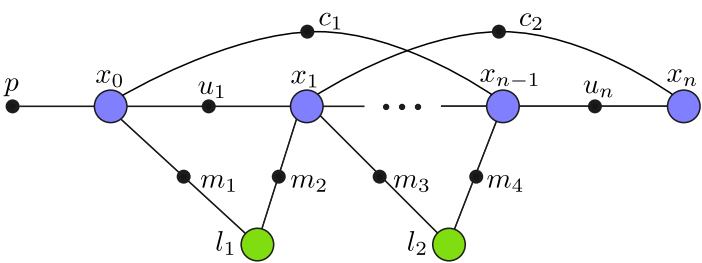

Fig. 2. The factor graph formulation of the full (smoothing) SLAM problem. Here variable nodes are shown as large circles and factor nodes as small solid circles. The variables consist of robot poses $x$ and landmark positions $l$, and the factors are odometry measurements $u$, a prior $p$, loop closing constraints $c$ and landmark measurements $m$. Each of the factors corresponds to a summand $r_{i}$ in (1). In the online case, as the robot explores previously unseen areas, new variable nodes (i.e. robot positions and landmarks) and factor nodes (measurements) are added to this graph over time; the corresponding online inference problem is then given by (2).

ubiquity of these models, robust and computationally efficient methods for solving (1) are thus of significant practical import.

In the case of online inference, the input data is collected sequentially, and we wish to obtain a revised estimate for $X$ after the incorporation of each datum. Since each input datum gives rise to a summand in (1), online inference corresponds to solving the sequence of sparse least-squares problems

$$
\begin{gathered}
\min _{x_{t} \in \mathbb{R}^{n_{t}}} S^{(t)}\left(x_{t}\right) \\
S^{(t)}\left(x_{t}\right)=\sum_{i=1}^{m_{t}} r_{i}\left(x_{i}\right)^{2}=\left\|r^{(t)}\left(x_{t}\right)\right\|^{2}
\end{gathered}
$$

for $r^{(t)}: \mathbb{R}^{n_{t}} \rightarrow \mathbb{R}^{m_{t}}$ and $t=1,2, \ldots$, where:

1) $m_{t}, n_{t} \in \mathbb{N}^{+}$are monotonically non-decreasing in $t$,

2) $m_{t} \geq n_{t}$ for all $t$

3) $x_{i} \in \mathbb{R}^{n_{i}}$ for all $i$ and $x_{i} \subseteq x_{j}$ for all $i \leq j$.

Condition 1 above expresses the fact that the summation in (2) evolves over time only through the addition of new terms. Condition 2 is necessary in order for the minimization problem in (2) to have a unique solution. Condition 3 formalizes the idea that we also allow the vector of states $X$ that we wish to estimate to be augmented online by the addition of new quantities of interest (for example, as in the case of robotic mapping when exploring previously unseen areas, cf. Fig. 2).

Our goal in this paper is to develop a fully incremental algorithm capable of robustly solving online sparse leastsquares minimization problems of the form (2) in real-time.

\section{REVIEW OF NEWTON-TYPE OPTIMIZATION METHODS} AND ISAM

The RISE algorithm that we develop in Section V exploits iSAM's incremental computation of the Gauss-Newton step in order to solve the sequential sparse least-squares problem (2) efficiently in the online case. In this section, we review the general class of Newton-type optimization methods, their specialization to the Gauss-Newton method, and Gauss-Newton's incremental implementation in iSAM.

\section{A. Newton's method and its approximations}

Newton's method [8], [11] is an iterative numerical method for estimating a solution $x^{*}$ of the general nonlinear minimization problem

$$
\min _{x \in \mathbb{R}^{n}} f(x), \quad f \in C^{2}\left(\mathbb{R}^{n}\right) .
$$


Given an initial estimate $x^{(i)}$ for $x^{*}$, the function $f$ is locally approximated at $x^{(i)}$ by its second-order Taylor expansion $q^{(i)}$ :

$$
q^{(i)}\left(x^{(i)}+h\right)=f\left(x^{(i)}\right)+\nabla f\left(x^{(i)}\right)^{T} h+\frac{1}{2} h^{T} \frac{\partial^{2} f}{\partial x^{2}}\left(x^{(i)}\right) h,
$$

and a revised estimate

$$
x^{(i+1)}=x^{(i)}+h_{N}^{(i)}
$$

is computed by choosing the Newton step $h_{N}^{(i)}$ to be any increment to $x^{(i)}$ that minimizes the value of the local approximation (4):

$$
h_{N}^{(i)} \in \underset{h \in \mathbb{R}^{n}}{\operatorname{argmin}} q^{(i)}\left(x^{(i)}+h\right) .
$$

Provided that $\frac{\partial^{2} f}{\partial x^{2}}\left(x^{(i)}\right)>0$, there is a unique minimizer $h_{N}^{(i)}$ in (6), which is determined as the solution of

$$
\frac{\partial^{2} f}{\partial x^{2}}\left(x^{(i)}\right) h_{N}^{(i)}=-\nabla f\left(x^{(i)}\right) .
$$

Assuming that each of the steps $h_{N}^{(i)}$ in (6) exists, Newton's method consists of iteratively applying equations (4), (6), and (5), in that order, to generate a sequence of estimates $x^{(0)}, x^{(1)}, \ldots$ for $x^{*}$ until some stopping criterion is satisfied.

Newton's method has several attractive theoretical properties, in particular a fast (quadratic) convergence rate when initialized with an estimate $x^{(0)}$ that is close to a minimizer $x^{*}$ of a sufficiently regular function $f$ [8]. However, in application it may not always be practical or computationally feasible to evaluate the gradient $\nabla f\left(x^{(i)}\right)$ or Hessian $\frac{\partial^{2} f}{\partial x^{2}}\left(x^{(i)}\right)$ in the quadratic model (4) at every iteration (depending upon the dimension $n$ and analytical complexity of the function $f$ ). In these cases, the local model (4) is often relaxed to

$$
q^{(i)}\left(x^{(i)}+h\right)=f\left(x^{(i)}\right)+\left(g^{(i)}\right)^{T} h+\frac{1}{2} h^{T} B^{(i)} h,
$$

where $g^{(i)} \in \mathbb{R}^{n}$ and $B^{(i)} \in \mathbb{R}^{n \times n}$ symmetric are chosen such that

$$
g^{(i)} \approx \nabla f\left(x^{(i)}\right), \quad B^{(i)} \approx \frac{\partial^{2} f}{\partial x^{2}}\left(x^{(i)}\right),
$$

and the corresponding update step $h_{N}^{(i)}$ is computed as a solution of

$$
B^{(i)} h_{N}^{(i)}=-g^{(i)} .
$$

The selection of different methods for performing the approximations (9) gives rise to a broad class of optimization algorithms collectively referred to as Newton-type or approximate Newton methods. With a careful choice of approximation scheme in (9) it is possible to preserve many of the desirable properties of Newton's method (most importantly a superlinear end-stage convergence rate) while dramatically reducing the computational burden of computing the update steps $h_{N}^{(i)}$.

\section{B. The Gauss-Newton method}

The Gauss-Newton method [8], [11] is an approximate Newton method for solving the minimization problem (3) in the special case (1) in which the objective function is a sum of squared nonlinear terms. In this case we have

$$
\begin{aligned}
\frac{\partial S}{\partial x_{j}} & =2 \sum_{i=1}^{m} r_{i} \frac{\partial r_{i}}{\partial x_{j}} \\
\frac{\partial^{2} S}{\partial x_{j} \partial x_{k}} & =2 \sum_{i=1}^{m} \frac{\partial r_{i}}{\partial x_{j}} \frac{\partial r_{i}}{\partial x_{k}}+r_{i} \frac{\partial^{2} r_{i}}{\partial x_{j} \partial x_{k}},
\end{aligned}
$$

and the Gauss-Newton method is obtained as an approximate Newton method by ignoring the effects of the second-order partial derivatives of $r$ when forming the approximate Hessian $B^{(i)}$ in (9):

$$
\frac{\partial^{2} S}{\partial x_{j} \partial x_{k}} \approx 2 \sum_{i=1}^{m} \frac{\partial r_{i}}{\partial x_{j}} \frac{\partial r_{i}}{\partial x_{k}}
$$

(the exact gradient $\nabla S\left(x^{(i)}\right)$ corresponding to (11a) is used for $\left.g^{(i)}\right)$. Using the function $r: \mathbb{R}^{n} \rightarrow \mathbb{R}^{m}$, we can write this approximation more conveniently in matrix notation as

$$
\begin{aligned}
g^{(i)} & =2 J\left(x^{(i)}\right)^{T} r\left(x^{(i)}\right), \\
B^{(i)} & =2 J\left(x^{(i)}\right)^{T} J\left(x^{(i)}\right),
\end{aligned}
$$

where $J\left(x^{(i)}\right)$ denotes the Jacobian of $r$ evaluated at $x^{(i)}$ :

$$
J\left(x^{(i)}\right)=\left.\frac{\partial r}{\partial x}\right|_{x=x^{(i)}} \in \mathbb{R}^{m \times n} .
$$

We observe that the approximation (12) is equivalent to the assumption that $r$ is locally linear. Indeed, substitution of (13) into (8) produces

$$
\begin{aligned}
q^{(i)}\left(x^{(i)}+h\right)= & r\left(x^{(i)}\right)^{T} r\left(x^{(i)}\right)+2 r\left(x^{(i)}\right)^{T} J\left(x^{(i)}\right) h \\
& +h^{T} J\left(x^{(i)}\right)^{T} J\left(x^{(i)}\right) h \\
= & \left\|L^{(i)}\left(x^{(i)}+h\right)\right\|^{2},
\end{aligned}
$$

where

$$
L^{(i)}\left(x^{(i)}+h\right)=r\left(x^{(i)}\right)+J\left(x^{(i)}\right) h
$$

is the first-order Taylor expansion (i.e. linearization) of $r$ about $x^{(i)}$. Consequently, by virtue of (11b), (12), and (15), we expect the Gauss-Newton method to produce the best results when applied to functions $r$ that have relatively modest nonlinearities (as quantified by the magnitudes of their second partial derivatives) and small magnitudes $\|r\|$.

To compute the Gauss-Newton step $h_{G N}^{(i)}$, we observe that if $J\left(x^{(i)}\right)$ is full-rank, then $B^{(i)}$ as defined in (13b) is positive definite, and thus $h_{G N}^{(i)}$ is uniquely determined by

$$
2 J\left(x^{(i)}\right)^{T} J\left(x^{(i)}\right) h_{G N}^{(i)}=-2 J\left(x^{(i)}\right)^{T} r\left(x^{(i)}\right)
$$

following (10). Letting

$$
Q^{(i)}\left(\begin{array}{c}
R^{(i)} \\
0
\end{array}\right)=J\left(x^{(i)}\right)
$$

be a QR decomposition [19] of the Jacobian $J\left(x^{(i)}\right)$ and

$$
\left(\begin{array}{l}
d^{(i)} \\
e^{(i)}
\end{array}\right)=\left(Q^{(i)}\right)^{T} r\left(x^{(i)}\right)
$$

for $d^{(i)} \in \mathbb{R}^{n}$ and $e^{(i)} \in \mathbb{R}^{m-n}$, we can simplify (17) to

$$
R^{(i)} h_{G N}^{(i)}=-d^{(i)} .
$$

Since $R^{(i)}$ is upper-triangular, equation (20) can be efficiently solved for $h_{G N}^{(i)}$ by back-substitution. 


\section{C. iSAM: Incrementalizing Gauss-Newton}

As shown in Section II, the arrival of new data corresponds to augmenting the function $r=r_{\text {old }}: \mathbb{R}^{n} \rightarrow \mathbb{R}^{m}$ on the righthand side of (1) to the function

$$
\begin{aligned}
\bar{r}: \mathbb{R}^{n+n_{\text {new }}} & \rightarrow \mathbb{R}^{m+m_{\text {new }}} \\
\bar{r}\left(x_{\text {old }}, x_{\text {new }}\right) & =\left(\begin{array}{c}
r_{\text {old }}\left(x_{\text {old }}\right) \\
r_{\text {new }}\left(x_{\text {old }}, x_{\text {new }}\right)
\end{array}\right),
\end{aligned}
$$

where here $r_{\text {new }}: \mathbb{R}^{n+n_{\text {new }}} \rightarrow \mathbb{R}^{m_{\text {new }}}$ is the set of new measurement functions and $x_{n e w} \in \mathbb{R}^{n_{n e w}}$ is the set of new system variables introduced as a result of the new observations.

In the naïve application of the Gauss-Newton algorithm of Section III-B, the solution $x^{*}=\left(x_{\text {old }}^{*}, x_{n e w}^{*}\right)$ for the augmented least-squares problem determined by (21) would be found by performing Gauss-Newton iterations until convergence. However, in the context of the sequential estimation problem (2) we already have a good estimate $\hat{x}_{\text {old }}$ for the values of the previously-introduced variables $x_{\text {old }}$, obtained by solving the least-squares minimization problem (1) prior to the introduction of the new measurement functions $r_{n e w}$. Thus, given any good initialization $\hat{x}_{n e w}$ for the newly-introduced system variables $x_{n e w}, \hat{x}=\left(\hat{x}_{\text {old }}, \hat{x}_{\text {new }}\right)$ provides a good initialization for the augmented state $x=\left(x_{\text {old }}, x_{n e w}\right)$ for the Gauss-Newton algorithm.

Furthermore, since we generally expect the initial estimate $\hat{x}$ to be close to the true minimizing value $x^{*}$, it is not usually necessary to iterate the Gauss-Newton algorithm until convergence after integration of every new observation; instead, a single Gauss-Newton step is computed and used to correct the initial estimate $\hat{x}$. The advantage to this approach is that it avoids having to recompute the Jacobian $\bar{J}(\hat{x})$ for $\bar{r}$ and its QR decomposition anew each time new observations arrive; instead, iSAM efficiently obtains $\bar{J}(\hat{x})$ together with its QR decomposition by updating the Jacobian $J\left(\hat{x}_{\text {old }}\right)$ and its QR decomposition, as we now describe.

Letting $x=\left(x_{\text {old }}, x_{n e w}\right)$, the Jacobian $\bar{J}(x)$ for the augmented system (21) can be decomposed into block form as

$$
\bar{J}(x)=\frac{\partial \bar{r}}{\partial x}=\left(\begin{array}{cc}
\frac{\partial r_{\text {old }}}{\partial x_{\text {old }}} & 0 \\
\frac{\partial r_{\text {new }}}{\partial x_{\text {old }}} & \frac{\partial r_{\text {new }}}{\partial x_{\text {new }}}
\end{array}\right)=\left(\begin{array}{cc}
J\left(x_{\text {old }}\right) & 0 \\
J_{\text {new }}(x)
\end{array}\right)
$$

where

$$
J\left(x_{\text {old }}\right)=\frac{\partial r_{\text {old }}}{\partial x_{\text {old }}} \in \mathbb{R}^{m \times n}
$$

is the Jacobian of the previous function $r_{\text {old }}$ and

$$
J_{\text {new }}(x)=\frac{\partial r_{n e w}}{\partial x} \in \mathbb{R}^{m_{n e w} \times\left(n+n_{n e w}\right)}
$$

is the Jacobian of the new measurement function $r_{n e w}$. Letting

$$
J\left(\hat{x}_{\text {old }}\right)=\left(\begin{array}{ll}
Q_{1} & Q_{2}
\end{array}\right)\left(\begin{array}{c}
R \\
0
\end{array}\right)
$$

be a QR decomposition for $J\left(\hat{x}_{\text {old }}\right)$, where $Q_{1} \in \mathbb{R}^{m \times n}$ and $Q_{2} \in \mathbb{R}^{m \times(m-n)}$, we have

$$
\left(\begin{array}{ccc}
Q_{1} & 0 & Q_{2} \\
0 & I & 0
\end{array}\right)\left(\begin{array}{cc}
R & 0 \\
J_{\text {new }}(\hat{x}) \\
0
\end{array}\right)=\left(\begin{array}{cc}
Q_{1} R & 0 \\
J_{\text {new }}(\hat{x})
\end{array}\right)=\bar{J}(\hat{x})
$$

by (22) and (25), which gives a partial QR decomposition of $\bar{J}(\hat{x})$. This partial decomposition can be completed by using Givens rotations to zero out the remaining nonzero elements below the main diagonal. Let $G \in \mathbb{R}^{\left(n+m_{n e w}\right) \times\left(n+m_{n e w}\right)}$ denote a matrix of Givens rotations such that

$$
G\left(\begin{array}{cc}
R & 0 \\
J_{\text {new }}(\hat{x})
\end{array}\right)=\left(\begin{array}{c}
\bar{R} \\
0
\end{array}\right)
$$

where $\bar{R} \in \mathbb{R}^{\left(n+n_{\text {new }}\right) \times\left(n+n_{\text {new }}\right)}$ is upper-triangular. Defining

$$
\bar{G}=\left(\begin{array}{cc}
G & 0 \\
0 & I
\end{array}\right), \quad \bar{Q}=\left(\begin{array}{ccc}
Q_{1} & 0 & Q_{2} \\
0 & I & 0
\end{array}\right) \bar{G}^{T},
$$

(so that $\bar{G}, \bar{Q} \in \mathbb{R}^{\left(m+m_{n e w}\right) \times\left(m+m_{n e w}\right)}$ are also orthogonal), equations (26), (27) and (28) show that

$$
\bar{J}(\hat{x})=\bar{Q}\left(\begin{array}{c}
\bar{R} \\
0
\end{array}\right)
$$

is a QR decomposition for the augmented Jacobian $\bar{J}(\hat{x})$. Now we can use (19) and (20) to compute the Gauss-Newton step $\bar{h}_{G N}$ for the augmented system:

$$
\begin{aligned}
\bar{Q}^{T} \bar{r}(\hat{x}) & =\left(\begin{array}{cc}
G & 0 \\
0 & I
\end{array}\right)\left(\begin{array}{cc}
Q_{1}^{T} & 0 \\
0 & I \\
Q_{2}^{T} & 0
\end{array}\right)\left(\begin{array}{c}
r_{\text {old }}\left(\hat{x}_{\text {old }}\right) \\
r_{\text {new }}(\hat{x})
\end{array}\right) \\
& =\left(\begin{array}{ll}
G & 0 \\
0 & I
\end{array}\right)\left(\begin{array}{c}
d_{\text {old }} \\
r_{\text {new }}(\hat{x}) \\
e_{\text {old }}
\end{array}\right) \\
& =\left(\begin{array}{c}
\bar{d} \\
e_{\text {new }} \\
e_{\text {old }}
\end{array}\right) \\
& =\left(\begin{array}{c}
\bar{d} \\
\bar{e}
\end{array}\right)
\end{aligned}
$$

where

$$
\left(\begin{array}{c}
\bar{d} \\
e_{\text {new }}
\end{array}\right)=G\left(\begin{array}{c}
d_{\text {old }} \\
r_{\text {new }}(\hat{x})
\end{array}\right)
$$

for $\bar{d} \in \mathbb{R}^{n+n_{n e w}}$. The Gauss-Newton step $\bar{h}_{G N}$ used to correct the estimate $\hat{x}$ for the augmented system is then computed as the solution of

$$
\bar{R} \bar{h}_{G N}=-\bar{d} \text {. }
$$

Equations (24), (27) and (31) show how to obtain the $\bar{R}$ factor of the QR decomposition of $\bar{J}(\hat{x})$ and the corresponding linear system (32) by updating the $R$ factor and linear system for the previous Jacobian $J\left(\hat{x}_{\text {old }}\right)$ using Givens rotations. Since the updated factor $\bar{R}$ and the new right-hand side vector $\bar{d}$ are obtained by applying $G$ directly to the augmented factor $R$ in (27) and the augmented right-hand side vector $d$ in (31), it is not necessary to explicitly form the orthogonal matrix $\bar{Q}$ in (28). Nor is it necessary to form the matrix $G$ explicitly either; instead, the appropriate individual Givens rotations can be applied sequentially directly to the matrix in (27) and the righthand side vector in (31). Under the assumption of sparsity, only a few elements of the the Jacobian $J_{\text {new }}(\hat{x})$ will be nonzero, and therefore only a small number of Givens rotations will be needed to perform the update in (27). Obtaining the linear system (32) by this method is thus a computationally efficient operation. 


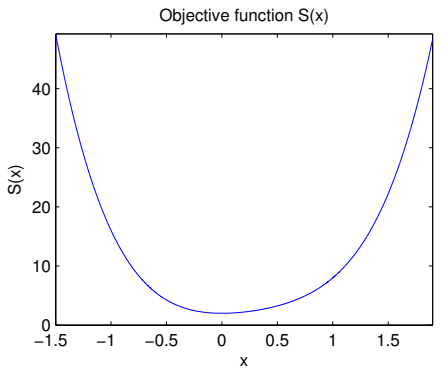

(a) The objective function $S(x)$

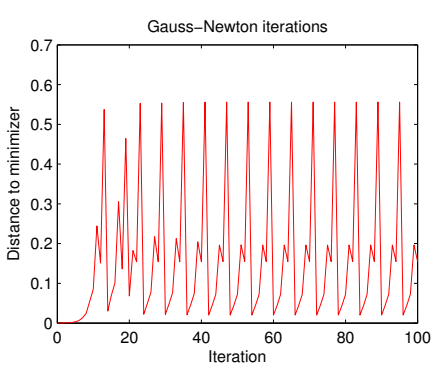

(b) Gauss-Newton iterates

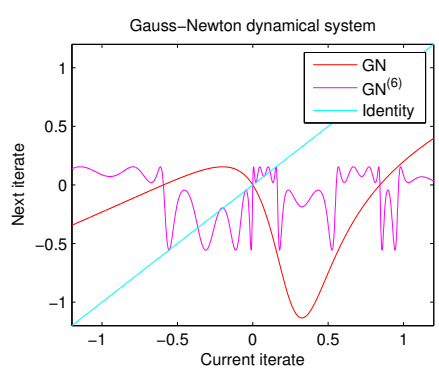

(c) Gauss-Newton dynamical system

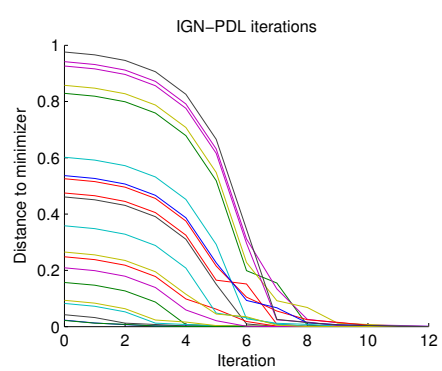

(d) IGN-PDL iterates

Fig. 3. Failure of the Gauss-Newton method. (a): The graph of the sum-of-squares objective function $S(x)$ determined by (33) for $\lambda=-2$. This function is $C^{\infty}$ and strictly convex, and has a unique global minimizer at $x^{*}=0$ with $\frac{\partial^{2} S}{\partial x^{2}}\left(x^{*}\right)>0$. (b): This plot shows the distance from the $i$ th Gauss-Newton iterate $x^{(i)}$ to the global minimizer $x^{*}$ for the first 100 iterations of a sequence initialized with $x^{(0)}=10^{-4}$. Note that the Gauss-Newton method initially drives the estimate $x^{(i)}$ away from the global minimum $x^{*}$ before converging to a 6-periodic orbit. (c): This figure illustrates the behavior of the Gauss-Newton update rule $G N: x^{(i)} \mapsto x^{(i+1)}$ and its 6-fold composition $G N^{(6)}$ considered as dynamical systems. The graph of $G N$ intersects the graph of the identity mapping at the minimizer $x^{*}=0$, showing that $x^{*}$ is a fixed point of $G N$ (as we would hope); however $\nabla G N\left(x^{*}\right)=-2$, so that $\left|\nabla G N\left(x^{*}\right)-1\right|=3>1$, and therefore this fixed point is repelling [20, Chp. 10]. The intersections of the graph of $G N^{(6)}$ with the graph of the identity give the 6-periodic points of $G N$, including the members of the 6-periodic orbit to which the Gauss-Newton iterates converge in (b). This example illustrates the Gauss-Newton method's sensitivity to nonlinearity even when applied to well-behaved functions with good initialization. (d): In contrast, the IGN-PDL algorithm of Section IV-C is globally convergent when applied to this function (cf. Theorem 3). This plot shows the distance from the $i$ th IGN-PDL iterate $x^{(i)}$ to the minimizer $x^{*}$ for 20 sequences with initializations $x^{(0)}$ sampled uniformly randomly from $[-1,1]$ and parameters $\Delta^{(0)}=.01, \eta_{1}=.25, \eta_{2}=.75, \gamma_{1}=.5, \gamma_{2}=2$. The 12 th iterates $x^{(12)}$ of these sequences are all within $3 \times 10^{-4}$ of the minimizer $x^{*}$. Strong global convergence properties and robustness to highly nonlinear and ill-conditioned systems are general features of this algorithm (cf. Sec. IV-D).

Finally, we observe that while relinearization is not needed after every new observation, the system should be periodically relinearized about its corrected estimate in order to perform a full Gauss-Newton iteration and obtain a better estimate of the local minimum (this is particularly true after observations which are likely to significantly alter the estimates of system variables). When relinearizing the system about the corrected estimate, the incremental updating method outlined above is no longer applicable; instead, the QR factorization of the Jacobian needs to be recomputed anew. While this is an expensive batch operation, the factorization step can be combined with a variable reordering step [21] in order to reduce the fill-in in the resulting factor $R$, thereby maintaining sparsity and speeding up subsequent incremental computations.

\section{From Gauss-Newton to Powell’s Dog-LeG}

The incrementalized Gauss-Newton method outlined in Section III-C is computationally efficient, straightforward to implement, and enjoys rapid (up to quadratic [11, pg. 113]) convergence near the minimum. However, the assumption of the local linearity of $r$ (which underlies the approximations (13b) and (15)) means that the Gauss-Newton method can exhibit poor behavior when either $r$ itself or its second partial derivatives $\frac{\partial^{2} r}{\partial x_{j} \partial x_{k}}$ have large magnitude. Indeed, convergence of the Gauss-Newton method is not guaranteed, not even locally; this is in marked contrast with the behavior of Newton's method, which (although not globally convergent) can at least guarantee fast local convergence for a class of functions that is not excessively restrictive in practice (cf. Theorem 3.5 of [8]). Furthermore, it is not difficult to construct simple (even quadratic) examples of $r$ where the sequence of Gauss-Newton iterates $\left\{x^{(i)}\right\}_{i=1}^{\infty}$ is in fact globally divergent (i.e. fails to converge when initialized with any point $x^{(0)} \neq x^{*}$ ). For example, consider the minimization (1) determined by

$$
\begin{aligned}
r & : \mathbb{R} \rightarrow \mathbb{R}^{2} \\
r(x) & =\left(\begin{array}{c}
x+1 \\
\lambda x^{2}+x-1
\end{array}\right) .
\end{aligned}
$$

For $\lambda=-2$, the function $S(x)$ is $C^{\infty}$ and strictly convex, and has a single global minimum at $x^{*}=0$ with $\frac{\partial^{2} S}{\partial x^{2}}\left(x^{*}\right)>0$, hence satisfies all of the standard regularity conditions customarily assumed in numerical optimization (cf. e.g. [8], [11]). Nevertheless, the Gauss-Newton algorithm is in fact globally divergent in this case [11,pg. 113]. This lack of robustness even under ideal conditions (a well-behaved objective function with strong regularity properties and good initialization, cf. Fig. 3) is a serious shortcoming of the Gauss-Newton method.

To address this shortcoming, in this paper we adopt the Powell's Dog-Leg algorithm [8], [12] as the method of choice for performing the sparse least-squares minimization (1). This algorithm combines the superlinear end-stage convergence speed of the Newton-type methods with the excellent global convergence properties [13]-[15] of gradient descent approaches. Indeed, when applied to sparse least-squares minimization problems, Powell's Dog-Leg performs significantly faster than Levenberg-Marquardt (the current method of choice in the robotics and computer vision communities) while maintaining comparable levels of accuracy [16].

\section{A. The trust-region approach}

As shown in Section III-A, in each iteration Newton's method constructs a local model $q^{(i)}$ for the objective function $f$ on a neighborhood $U^{(i)}$ of the current estimate $x^{(i)}$, and then determines an update step $h_{N}^{(i)}$ that minimizes $q^{(i)}$ in place of $f$. However, the model $q^{(i)}$ in (4) is constructed using information about $f$ 's first- and second-order derivatives at $x^{(i)}$, which depend only upon the local behavior of $f$ near $x^{(i)}$. 
Consequently, while $q^{(i)}$ is a good approximation of $f$ near $x^{(i)}$ (in a sense made precise by Taylor's Theorem), its global behavior may be quite different from $f$ 's. This can become problematic if the update step $h_{N}^{(i)}$ computed using $q^{(i)}$ leaves the region $U^{(i)}$ in which $f$ is well-approximated by $q^{(i)}$.

Trust-region methods [8] address this hazard by maintaining an explicit trust-region, an open ball of radius $\Delta^{(i)}$ centered on the current estimate $x^{(i)}$ within which $f$ is considered well-approximated by the local model $q^{(i)}$. The trust-region update step $h_{t r}^{(i)}$ is then obtained as an increment to $x^{(i)}$ that minimizes the value of $q^{(i)}$, subject to the condition that the update step does not leave the trust-region:

$$
h_{t r}^{(i)} \in \underset{\|h\| \leq \Delta^{(i)}}{\operatorname{argmin}} q^{(i)}\left(x^{(i)}+h\right) .
$$

The following theorem gives necessary and sufficient optimality conditions characterizing the trust-region step $h_{t r}^{(i)}$ in (34) when $q^{(i)}$ is a quadratic model (as in approximate Newton methods). Its proof follows from a straightforward application of the Karush-Kuhn-Tucker first-order optimality conditions together with the enforcement of the necessary second-order conditions for a minimizer (cf. [8, Chps. 4 and 12]).

Theorem 1 (Optimality conditions for the trust-region step). Let $f, g \in \mathbb{R}^{n}$, let $B \in \mathbb{R}^{n \times n}$ be symmetric, and define

$$
\begin{gathered}
q: \mathbb{R}^{n} \rightarrow \mathbb{R} \\
q(h)=f+g^{T} h+\frac{1}{2} h^{T} B h .
\end{gathered}
$$

Then $h^{*} \in \mathbb{R}^{n}$ is a solution of the constrained minimization

$$
\min _{h \in \mathbb{R}^{n}} q(h) \quad \text { s.t. }\|h\| \leq \Delta
$$

for $\Delta>0$ if and only if $\left\|h^{*}\right\| \leq \Delta$ and there exists $\lambda^{*} \geq 0$ such that the following conditions are satisfied:

$$
\begin{aligned}
\left(B+\lambda^{*} I\right) h^{*} & =-g, \\
\lambda^{*}\left(\Delta-\left\|h^{*}\right\|\right) & =0, \\
\left(B+\lambda^{*} I\right) & \geq 0 .
\end{aligned}
$$

As the trust-region method proceeds, the radius of the trust region $\Delta^{(i)}$ is varied adaptively according to the gain ratio:

$$
\rho^{(i)}=\frac{\operatorname{ared}^{(i)}\left(h_{t r}^{(i)}\right)}{\operatorname{pred}^{(i)}\left(h_{t r}^{(i)}\right)},
$$

where

$$
\begin{aligned}
& \operatorname{ared}^{(i)}\left(h_{t r}^{(i)}\right)=f\left(x^{(i)}\right)-f\left(x^{(i)}+h_{t r}^{(i)}\right), \\
& \operatorname{pred}^{(i)}\left(h_{t r}^{(i)}\right)=q^{(i)}\left(x^{(i)}\right)-q^{(i)}\left(x^{(i)}+h_{t r}^{(i)}\right) ;
\end{aligned}
$$

this compares the actual reduction in the objective function's value obtained by taking the proposed trust-region step $h_{t r}^{(i)}$ with the predicted reduction in function value using the local model $q^{(i)}$. Values of $\rho$ close to 1 indicate that the local model $q^{(i)}$ is performing well near the current iterate $x^{(i)}$, so the trust-region radius $\Delta^{(i)}$ can be increased in the next iteration (to allow longer steps and hence faster convergence), while values close to 0 indicate that the local model is performing poorly, and $\Delta^{(i)}$ should be reduced accordingly (Algorithm 1). The entire trust-region method is summarized as Algorithm 2;
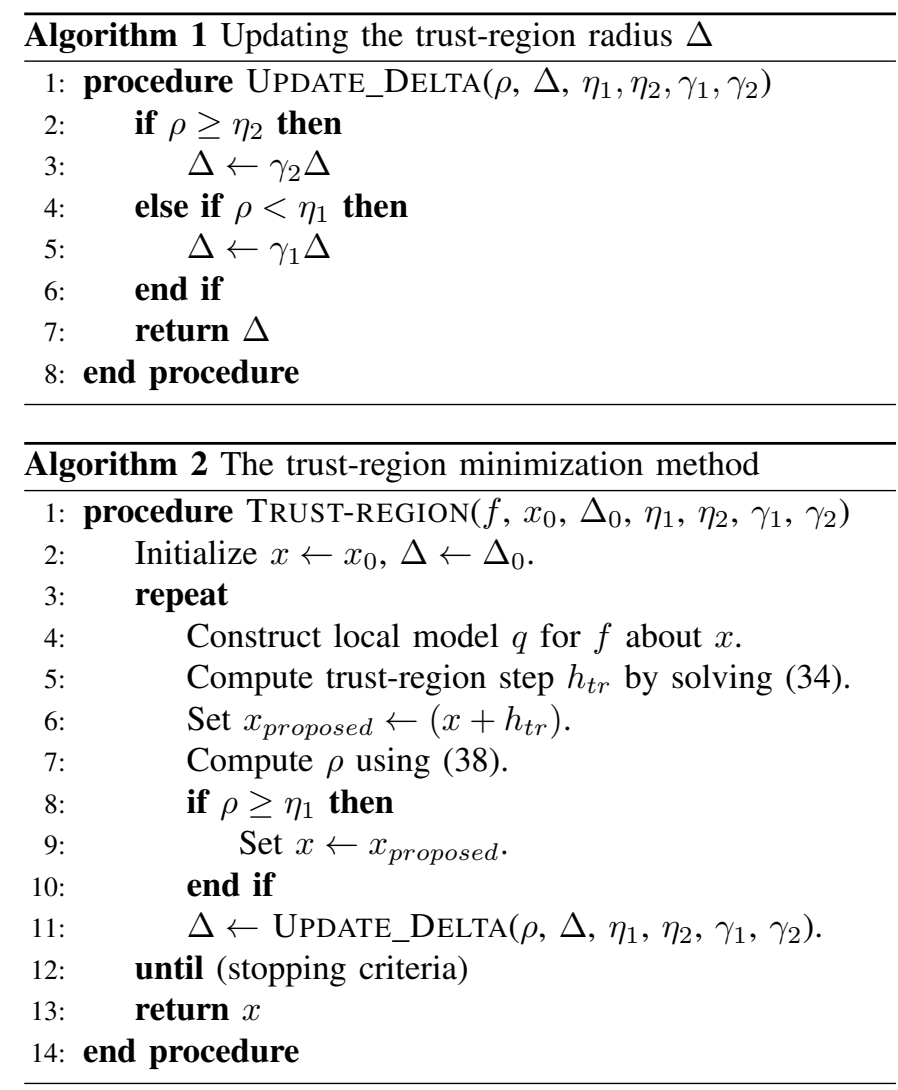

here $0<\eta_{1}<\eta_{2}<1$ and $0<\gamma_{1}<1<\gamma_{2}$ are usersupplied parameters specifying the gain-ratio thresholds and scaling factors used in the update of the trust-region radius.

\section{B. Powell's Dog-Leg}

While the canonical trust-region method (Algorithm 2) is intellectually pleasing and enjoys excellent convergence and robustness properties (cf. Section IV-D), it is not commonly used in practice because of the computational cost of finding the exact trust-region step $h_{t r}$ in (34). (Briefly, this entails solving a root-finding problem for the Lagrange multiplier $\lambda^{*}$ in (37a) that generally requires multiple factorizations of matrices of the form $B+\lambda I$ [22], a prohibitively expensive operation when appearing as a single step within another iterative algorithm.) Instead, most practical trust-region implementations solve (34) approximately using a computationally cheap approximation that is nevertheless accurate enough to preserve the desirable properties of the canonical algorithm. In this section we describe Powell's Dog-Leg [8], [12], one such approximate method that can be used whenever the matrix $B$ in the local quadratic model $q$ in (34) is positive definite.

The dog-leg approximation is derived by regarding the trustregion step computed in (34) as a function $h_{t r}(\Delta)$ of the trustregion radius and considering the effect of varying $\Delta$. For $B \in \mathbb{R}^{n \times n}$ symmetric and positive definite, the local model $q$ has a unique unconstrained minimizer: the Newton step

$$
h_{N}=-B^{-1} g \text {. }
$$

If the trust-region radius $\Delta$ is greater than the length of this step, then the constraint in (34) is inactive, and therefore

$$
h_{t r}(\Delta)=h_{N}, \quad\left\|h_{N}\right\| \leq \Delta .
$$


Conversely, when the trust-region radius $\Delta$ is small, the (second-order) quadratic term in the local model (8) is dominated by the linear term, and therefore the trust-region step computed in (34) will be well-approximated by the maximum step length allowable in the direction of steepest descent:

$$
h_{t r}(\Delta) \approx-\frac{\Delta}{\|g\|} g, \quad \Delta \text { small. }
$$

As $\Delta$ increases through the range $[0, \infty)$, the exact trustregion step $h_{t r}(\Delta)$ traces a smooth path interpolating these cases; Powell's Dog-Leg approximates this path using a piecewise linear path with two segments (Fig. 4). The first segment extends from $x$ to the gradient descent step $h_{g d}$, which we define as the unconstrained minimizer of the local model $q$ along the steepest descent direction:

$$
h_{g d}=-\alpha g, \quad \alpha=\underset{a \in \mathbb{R}^{+}}{\operatorname{argmin}} q(x-a g) .
$$

Using the definition of the local model (8) and the fact that $B>0$ by hypothesis, the gradient descent step defined in (43) can be written in closed-form as:

$$
h_{g d}=-\alpha g, \quad \alpha=\frac{g^{T} g}{g^{T} B g} .
$$

The second segment linearly interpolates the gradient descent step $h_{g d}$ and the Newton step $h_{N}$. Parameterizing the dog-leg path as $p_{d l}:[0,1] \rightarrow \mathbb{R}^{n}$, we have

$$
p_{d l}(t)= \begin{cases}2 t h_{g d}, & 0 \leq t \leq \frac{1}{2}, \\ h_{g d}+(2 t-1)\left(h_{N}-h_{g d}\right), & \frac{1}{2} \leq t \leq 1 .\end{cases}
$$

A direct computation using the definitions (40), (43), and (45) proves the following result (cf. e.g. [8, Sec. 4.1]).

Lemma 1. The dog-leg path $p_{d l}$ defined in (45) satisfies the following properties:

(i) $\left\|p_{d l}(t)\right\|$ is monotonically increasing for all $t \in[0,1]$.

(ii) $q\left(p_{d l}(t)\right)$ is monotonically decreasing for all $t \in[0,1]$.

By virtue of Lemma 1, the Powell's Dog-Leg step $h_{d l}$ is defined to be the (unique) farthest point along the dog-leg path $p_{d l}$ lying inside the trust-region boundary (cf. Fig. 1):

$$
\begin{aligned}
h_{d l} & =p_{d l}(\tau), \\
\tau & =\max \left\{t \in[0,1] \mid\left\|p_{d l}(t)\right\| \leq \Delta\right\} .
\end{aligned}
$$

The algorithmic computation of the dog-leg step $h_{d l}$ corresponding to definition (46) is given in Algorithm 3. The scalar $\beta$ appearing in line 7 is chosen to satisfy

$$
\left\|h_{g d}+\beta\left(h_{N}-h_{g d}\right)\right\|^{2}=\Delta^{2},
$$

which is quadratic in $\beta$. By Lemma 1 , (47) has exactly one solution in $(0,1)$, which can be written in closed form as

$$
\begin{aligned}
& v=h_{N}-h_{g d}, \\
& \beta=\frac{-h_{g d}^{T} v+\sqrt{\left(h_{g d}^{T} v\right)^{2}+\left(\Delta^{2}-\left\|h_{g d}\right\|^{2}\right)\|v\|^{2}}}{\|v\|^{2}} .
\end{aligned}
$$

The complete Powell's Dog-Leg algorithm is obtained from Algorithm 2 by replacing the computation of the trust-region step $h_{t r}$ in line 5 with the computation of the dog-leg step $h_{d l}$ defined by equations (40), (44), and Algorithm 3.

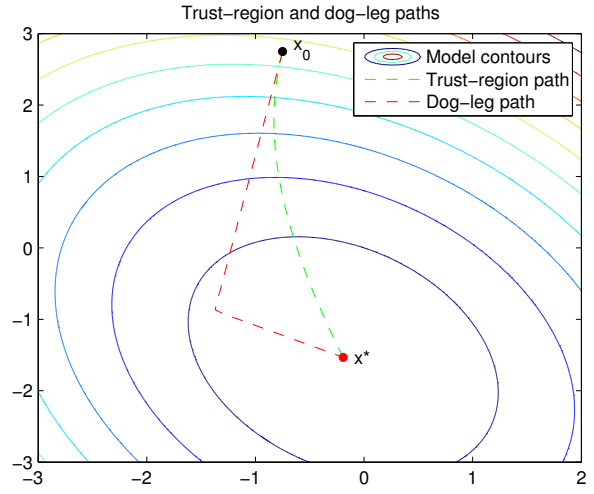

Fig. 4. This figure shows the trust-region and Powell's Dog-Leg paths for a quadratic model $q$ constructed at $x_{0}$ with minimizer $x^{*}$. As the trustregion radius $\Delta$ increases, the trust-region step $h_{t r}(\Delta)$ traces a smooth path interpolating the constrained gradient descent step and the Newton step $h_{N}$. Powell's Dog-Leg approximates this path using a piecewise linear path with two segments: the first from $x_{0}$ to the full gradient descent step $h_{g d}$, and the second from $h_{g d}$ to the Newton step $h_{N}$.

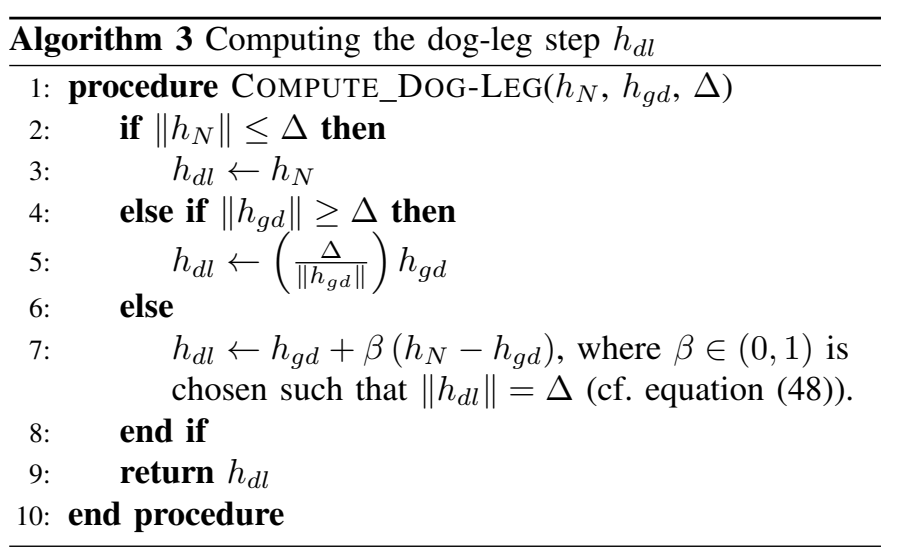

\section{Indefinite Gauss-Newton-Powell's Dog-Leg}

In this subsection we derive an approximate trust-region algorithm based on Powell's Dog-Leg with Gauss-Newton steps for solving minimization problems of the form (1).

To begin, we observe that by virtue of the Hessian approximations (13b) used in the Gauss-Newton local model $q, B \geq 0$ always. If $J(x)$ is full-rank, then $B>0$ and the dog-leg step $h_{d l}$ defined in Section IV-B exists and can be computed in terms of the Gauss-Newton step $h_{G N}$ and the gradient descent step $h_{g d}$. Equations (18)-(20) already provide a closed-form solution for computing the Gauss-Newton step, so it suffices to provide a closed-form solution for the gradient descent step $h_{g d}$ in (44). Substituting the expressions for $B$ and $g$ from (13) into (44), we find

$$
g=2 J(x)^{T} r(x), \quad \alpha=\frac{\|g\|^{2}}{2\|J(x) g\|^{2}} .
$$

Equations (18)-(20), (49), and Algorithm 3 thus enable the computation of the dog-leg step $h_{d l}$ when $B>0$.

In the case that $J(x)$ is not full-rank, $B$ is not positive definite and the dog-leg step is not defined. However, the Cauchy step $h_{C}$ (the constrained minimizer of the local model 


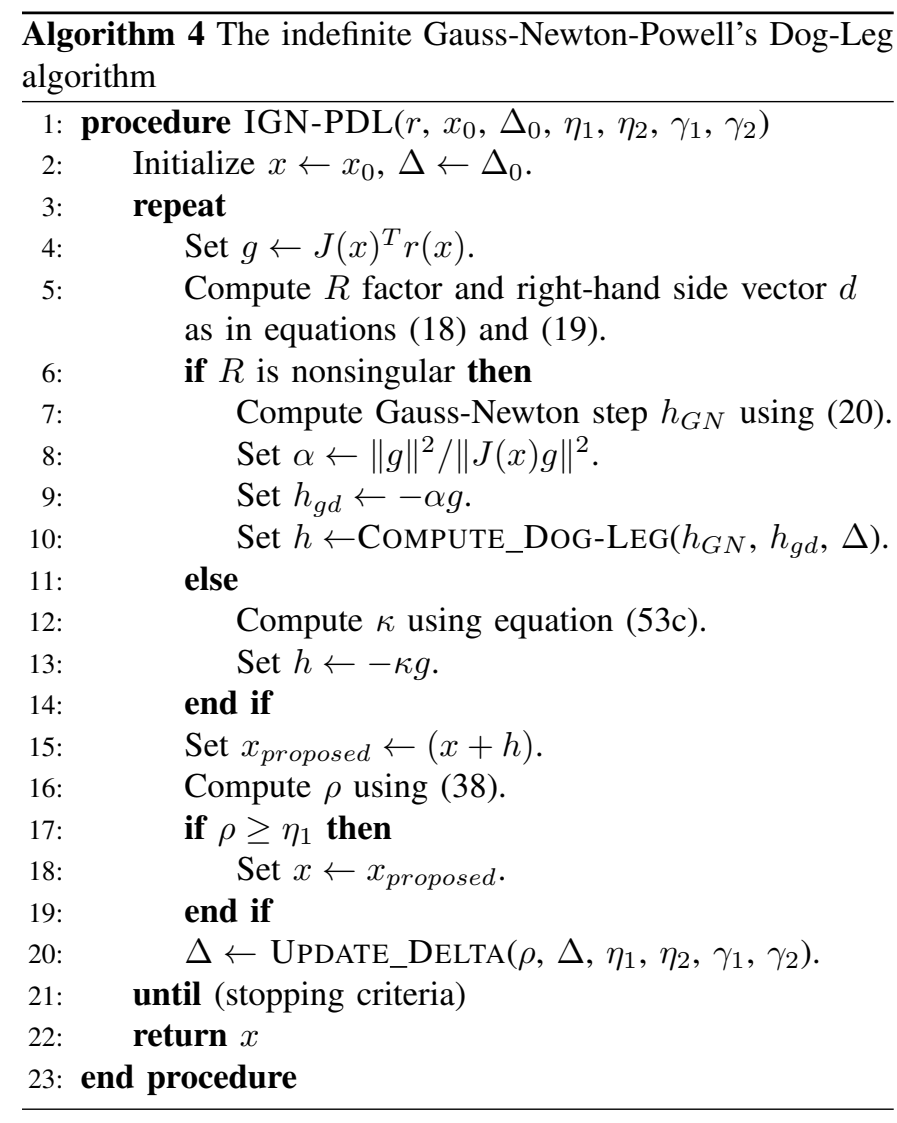

$q$ along the steepest descent direction):

$$
\begin{aligned}
h_{C} & =-\kappa g \\
\kappa & =\underset{0 \leq a \leq \frac{\Delta}{\|g\|}}{\operatorname{argmin}} q(x-a g)
\end{aligned}
$$

always exists and is unique for $g \neq 0$ and arbitrary symmetric $B \in \mathbb{R}^{n \times n}$ in the local model (8). Indeed, direct computation in (50) shows that the step-length $\kappa$ can be written in a simple closed form as:

$$
\kappa= \begin{cases}\min \left\{\frac{\Delta}{\|g\|}, \frac{\|g\|^{2}}{g^{T} B g}\right\}, & g^{T} B g>0 \\ \frac{\Delta}{\|g\|}, & g^{T} B g \leq 0\end{cases}
$$

Substituting the approximate Hessian (13b) from the GaussNewton local model into (51) produces:

$$
\kappa= \begin{cases}\min \left\{\frac{\Delta}{\|g\|}, \frac{\|g\|^{2}}{2\|J(x) g\|^{2}}\right\}, & \|J(x) g\|>0 \\ \frac{\Delta}{\|g\|}, & \|J(x) g\|=0\end{cases}
$$

Our proposed algorithm, which we refer to as the indefinite Gauss-Newton-Powell's Dog-Leg (IGN-PDL) algorithm, is obtained from Algorithm 2 by using Gauss-Newton-Powell's Dog-Leg steps in line 5 when $J(x)$ is full-rank, and constrained Cauchy steps otherwise.

Finally, for the sake of notational simplicity and to avoid unnecessary work, we simplify the computations in (49) and (52) by canceling the common factors of 2 in the gradient vector $g$ and the stepsizes $\alpha$ and $\kappa$. This produces

$$
\begin{aligned}
g & =J(x)^{T} r(x), \\
\alpha & =\frac{\|g\|^{2}}{\|J(x) g\|^{2}}, \\
\kappa & = \begin{cases}\min \left\{\frac{\Delta}{\|g\|}, \frac{\|g\|^{2}}{\|J(x) g\|^{2}}\right\}, & \|J(x) g\|>0, \\
\frac{\Delta}{\|g\|}, & \|J(x) g\|=0 .\end{cases}
\end{aligned}
$$

The IGN-PDL algorithm is summarized as Algorithm 4.

\section{Theoretical analysis}

While the trust-region approach of Section IV-A may appear to be only a slight variation on the Newton-type methods, in fact trust-region algorithms possess markedly superior robustness properties. In this subsection we examine two such properties (global convergence and robustness to numerical illconditioning), and prove a strong global convergence result for the IGN-PDL algorithm of Section IV-C.

1) Global convergence: One of the most attractive and powerful features of trust-region algorithms is that they are globally convergent under very general conditions, even when using (possibly very loose) gradient and Hessian approximations in the local models $q^{(i)}$. By way of illustration, the following remarkable theorem is due to R.G. Carter [13].

Theorem 2 (Global convergence of trust-region methods). Let $f: \mathbb{R}^{n} \rightarrow \mathbb{R}, x^{(0)} \in \mathbb{R}^{n}$, and let $\Omega \subseteq \mathbb{R}^{n}$ be any convex open set containing the sublevel set $\mathcal{L}_{f}\left(x^{(0)}\right)$ of $f$ at $x^{(0)}$ :

$$
\mathcal{L}_{f}\left(x^{(0)}\right)=\left\{x \in \mathbb{R}^{n} \mid f(x) \leq f\left(x^{(0)}\right)\right\} .
$$

Assume further that $f$ is lower-bounded on $\Omega, f \in C^{1}(\Omega)$, and that $\nabla f$ is Lipschitz continuous on $\Omega$.

Fix constants $0<\eta_{1}<\eta_{2}<1$ and $0<\gamma_{1}<1<\gamma_{2}$, $\zeta<1-\eta_{2}, \beta, \sigma \in(0, \infty)$, and $c \in(0,1]$, and let $\left\{x^{(i)}\right\}_{i=0}^{\infty}$ be the sequence of iterates obtained in Algorithm 2 using local models $q^{(i)}$ and approximate trust-region update steps $h_{t r}^{(i)}$ in line 5 that satisfy the following criteria:

(i) Feasibility: $\left\|h_{t r}^{(i)}\right\| \leq \Delta^{(i)}$ for all $i \geq 0$.

(ii) Gradient approximation error: the approximate gradients $g^{(i)}$ used in each of the local models $q^{(i)}$ satisfy the bounded relative error criterion:

$$
\frac{\left\|g^{(i)}-\nabla f\left(x^{(i)}\right)\right\|}{\left\|g^{(i)}\right\|} \leq \zeta .
$$

(iii) Uniform boundedness of approximate Hessians: each $B^{(i)} \in \mathbb{R}^{n \times n}$ is symmetric and satisfies $\left\|B^{(i)}\right\| \leq \beta$.

(iv) Asymptotic step direction: assuming that $\left\|B^{(i)}\right\| \leq \beta$ for all $i \geq 0$ and that

$$
\liminf _{i \rightarrow \infty}\left\|g^{(i)}\right\|>0 \text { and } \lim _{i \rightarrow \infty} \Delta^{(i)}=0,
$$

the step direction $\Theta^{(i)}$ satisfies

$$
\lim _{i \rightarrow \infty} \cos \Theta^{(i)}=1,
$$

where

$$
\cos \Theta^{(i)}=-\frac{\left(g^{(i)}\right)^{T} h_{t r}^{(i)}}{\left\|g^{(i)}\right\|\left\|h_{t r}^{(i)}\right\|} .
$$


(v) Uniform predicted decrease: each of the proposed update steps $h_{t r}^{(i)}$ satisfies:

$$
\operatorname{pred}^{(i)}\left(h_{t r}^{(i)}\right) \geq \frac{1}{2} c\left\|g^{(i)}\right\| \min \left\{\Delta^{(i)}, \frac{\left\|g^{(i)}\right\|}{\sigma}\right\} .
$$

Then either $\nabla f\left(x^{(i)}\right)=0$ for some iterate $x^{(i)}$, or the infinite subsequence $\left\{x^{\left(i_{k}\right)}\right\}_{k=0}^{\infty} \subseteq\left\{x^{(i)}\right\}_{i=0}^{\infty}$ of iterates that are accepted in line 9 of Algorithm 2 satisfies

$$
\lim _{k \rightarrow \infty}\left\|\nabla f\left(x^{\left(i_{k}\right)}\right)\right\|=0 .
$$

With the aid of Theorem 2, we prove the following convergence result for the IGN-PDL algorithm (Algorithm 4).

Theorem 3 (Global convergence of the IGN-PDL algorithm). Let $r: \mathbb{R}^{n} \rightarrow \mathbb{R}^{m}$ be $C^{2}$, define

$$
S(x)=\|r(x)\|^{2},
$$

and fix $0<\eta_{1}<\eta_{2}<1$ and $0<\gamma_{1}<1<\gamma_{2}$. Given any $x^{(0)} \in \mathbb{R}^{n}$, if the sublevel set $\mathcal{L}_{S}\left(x^{(0)}\right)$ is bounded, then the sequence of iterates $\left\{x^{(i)}\right\}$ accepted in line 18 of Algorithm 4 either terminates at some $x^{(k)}$ with $\nabla S\left(x^{(k)}\right)=0$ or satisfies

$$
\lim _{i \rightarrow \infty}\left\|\nabla S\left(x^{(i)}\right)\right\| \rightarrow 0 .
$$

This theorem is proved in the Appendix.

In practice, the hypotheses of Theorem 3 are quite general; intuitively, the bounded sublevel set condition simply prohibits the cost function $S$ from assigning the same quality to arbitrarily large regions of the state space. Any reasonable inferential cost function arising in practice will satisfy this condition.

2) Robustness to numerical ill-conditioning: In addition to their strong convergence properties, trust-region methods are also naturally robust against numerical ill-conditioning in the linear system (10) used to solve for the Newton step.

Recall that for a matrix $A \in \mathbb{R}^{m \times n}$ with $m \geq n$, the condition number $\kappa_{2}(A)$ (cf. [19, pg. 230]) is

$$
\kappa_{2}(A)= \begin{cases}\frac{\sigma_{\max }(A)}{\sigma_{\min }(A)}, & \operatorname{rank}(A)=n, \\ \infty, & \operatorname{rank}(A)<n,\end{cases}
$$

where $\sigma_{\max }(A)$ and $\sigma_{\min }(A)$ give the maximum and minimum singular values of $A$, respectively. Matrices $A$ with $\kappa_{2}(A) \gg 1$ are called ill-conditioned; these matrices tend to have poor numerical properties when used as the coefficient matrix for inverse problems of the form

$$
\min _{x \in \mathbb{R}^{n}}\|A x-b\|^{2}
$$

for $b \in \mathbb{R}^{m}$ (of which (10) and (15) are special cases). To see why this is so, observe that if $\operatorname{rank}(A)=n$, then (64) has the unique minimizer

$$
x_{L S}=\underset{x \in \mathbb{R}^{n}}{\operatorname{argmin}}\|A x-b\|^{2}=\sum_{i=1}^{n} \frac{u_{i}^{T} b}{\sigma_{i}} v_{i},
$$

where $\sigma_{1} \geq \cdots \geq \sigma_{n}>0$ are the singular values of $A$, and $u_{i} \in \mathbb{R}^{m \times 1}$ and $v_{i} \in \mathbb{R}^{n \times 1}$ are the left- and right-singular vectors (respectively) corresponding to $\sigma_{i}$ for all $1 \leq i \leq n$ (cf. [19, Sec. 5.5.3]). If $\kappa_{2}(A) \gg 1$, then since $\left\{u_{i}\right\}_{i=1}^{n}$ and $\left\{v_{i}\right\}_{i=1}^{n}$ are orthonormal sets, (65) shows that $x_{L S}$ will tend to be dominated by the effects of those (generally few) components of $b$ lying in subspaces spanned by left-singular vectors $u_{i}$ corresponding to small singular values $\sigma_{i}$. In practice, this commonly manifests as the least-squares solution $x_{L S}$ "exploding" (as measured by $\|\cdot\|$ ) through subspaces of $\mathbb{R}^{n}$ spanned by right-singular vectors $v_{i}$ corresponding to small singular values $\sigma_{i}$ whenever $A$ is ill-conditioned.

One standard approach for addressing poor numerical conditioning in (64) is Tikhonov regularization [23]. In this approach, the original problem (64) is replaced by

$$
\min _{x \in \mathbb{R}^{n}}\|A x-b\|^{2}+\lambda\|\Gamma x\|^{2},
$$

where $\Gamma \in \mathbb{R}^{n \times n}$ is a conditioning matrix designed to control some property of interest of the Tikhonov-regularized solution, and $\lambda \geq 0$ is a parameter controlling the applied degree of regularization. The minimizer $x_{\lambda}$ of (66) can be formally computed in closed form as

$$
\begin{aligned}
x_{\lambda} & =\underset{x \in \mathbb{R}^{n}}{\operatorname{argmin}}\|A x-b\|^{2}+\lambda\|\Gamma x\|^{2} \\
& =\left(A^{T} A+\lambda \Gamma^{T} \Gamma\right)^{-1} A^{T} b .
\end{aligned}
$$

The standard form of Tikhonov regularization has $\Gamma=I$, which simply controls the norm of $x_{\lambda}$. More interestingly, this choice also has the effect of improving the numerical conditioning of (66) versus (64). To see this, observe that (66) with $\Gamma=I$ can be expressed as an instance of (64):

$$
\min _{x \in \mathbb{R}^{n}}\|A x-b\|^{2}+\lambda\|x\|^{2}=\min _{x \in \mathbb{R}^{n}}\|\bar{A} x-\bar{b}\|^{2},
$$

where

$$
\bar{A}=\left(\begin{array}{c}
A \\
\sqrt{\lambda} I
\end{array}\right), \quad \bar{b}=\left(\begin{array}{l}
b \\
0
\end{array}\right) .
$$

The $n \times n$ block $\sqrt{\lambda} I$ of the augmented coefficient matrix $\bar{A}$ ensures that $\sigma_{\min }(\bar{A}) \geq \sqrt{\lambda}$, and the Pythagorean theorem implies that $\sigma_{\max }(\bar{A})=\|\bar{A}\| \leq \sqrt{\lambda+\|A\|^{2}}$. These two inequalities together imply that

$$
\kappa_{2}(\bar{A}) \leq \frac{\sqrt{\lambda+\|A\|^{2}}}{\sqrt{\lambda}}=\sqrt{1+\frac{\|A\|^{2}}{\lambda}} .
$$

Equation (70) shows that the regularization parameter $\lambda$ controls the effective conditioning of the Tikhonov-regularized system (66)-(69) when $\Gamma=I$.

We now highlight a remarkable connection between trustregion methods and Tikhonov regularization. Consider the optimality condition (37a) for the trust-region step in Theorem 1 , and suppose that $B \geq 0$. Since $B$ is symmetric, it can be diagonalized by an orthogonal matrix $Q$ :

$$
B=Q \operatorname{diag}\left(\lambda_{1}, \ldots, \lambda_{n}\right) Q^{T}
$$

where $\lambda_{1} \geq \cdots \geq \lambda_{n} \geq 0$ since $B \geq 0$; (71) is thus also a singular value decomposition for $B$, and $\kappa_{2}(B)=\lambda_{1} / \lambda_{n}$. The same $Q$ appearing in (71) also diagonalizes the matrix $\bar{B}=B+\lambda^{*} I$ appearing in (37a):

$$
\bar{B}=Q \operatorname{diag}\left(\lambda_{1}+\lambda^{*}, \ldots, \lambda_{n}+\lambda^{*}\right) Q^{T},
$$

and therefore

$$
\kappa_{2}(\bar{B})=\frac{\lambda_{1}+\lambda^{*}}{\lambda_{n}+\lambda^{*}} \leq \frac{\lambda_{1}}{\lambda_{n}}=\kappa_{2}(B),
$$


where the inequality in (73) is strict if $\lambda^{*}>0$.

Theorem 1 and equations (71)-(73) show that the trustregion step $h_{t r}$ determined by (34) can be interpreted as a kind of Tikhonov-regularized solution of the system (10) defining the (approximate) Newton step $h_{N}$; indeed, in the specific case of the Gauss-Newton system defined by (13), the corresponding first-order optimality condition (37a) is

$$
\left(J(x)^{T} J(x)+\lambda^{*} I\right) h_{t r}=-J(x)^{T} r(x),
$$

which is an instance of the Tikhonov-regularized system (67). In particular, equation (73) shows that $h_{t r}$ is the solution of a system whose conditioning is always at least as good as that of the system (10) defining $h_{N}$.

This analysis shows that trust-region methods are innately robust to ill-conditioning, and we therefore expect them to be particularly effective (as compared to pure Newton-type methods) whenever the approximate Hessians $B^{(i)}$ used in the local quadratic models (8) are ill-conditioned.

\section{RISE: INCREMENTALIZING PowelL's DoG-LeG}

In this section we present Robust Incremental least-Squares Estimation (RISE), an incrementalized version of the IGNPDL algorithm (Algorithm 4). For pedagogical clarity, we begin by following the original derivation of RISE as given in [24], in which IGN-PDL is incrementalized with the aid of iSAM. We then derive RISE2, a new and improved version of the RISE algorithm obtained by using iSAM2 [10] in place of the original iSAM.

\section{A. Derivation of RISE}

The IGN-PDL algorithm computes the approximate trustregion update step $h$ from the Gauss-Newton step $h_{G N}$ and the gradient descent step $h_{g d}$ when $R$ is nonsingular, and the Cauchy step $h_{C}$ when $R$ is singular (cf. lines 7-10 and 13 of Algorithm 4). As iSAM already implements an efficient incremental algorithm for computing $h_{G N}$ in the nonsingular case, it remains only to develop efficient methods to compute $h_{g d}$ and $h_{C}$. In turn, lines 9 and 13 of Algorithm 4 show that $h_{g d}$ and $h_{C}$ are computed in terms of the gradient direction vector $g$ and the scale factors $\alpha$ and $\kappa$ defined in (53). Thus, it suffices to determine efficient incrementalized versions of equations (53a)-(53c).

Letting $x=\left(x_{\text {old }}, x_{\text {new }}\right)$ as before and substituting the block decompositions (21) and (22) into (53a) produces

$$
\begin{aligned}
\bar{g} & =\bar{J}(\hat{x})^{T} \bar{r}(\hat{x}) \\
& =\left(\begin{array}{cc}
J\left(\hat{x}_{\text {old }}\right)^{T} & J_{\text {new }}(\hat{x})^{T} \\
0 &
\end{array}\right)\left(\begin{array}{c}
r_{\text {old }}\left(\hat{x}_{\text {old }}\right) \\
r_{\text {new }}(\hat{x})
\end{array}\right) \\
& =\left(\begin{array}{c}
J\left(\hat{x}_{\text {old }}\right)^{T} r_{\text {old }}\left(\hat{x}_{\text {old }}\right) \\
0
\end{array}\right)+J_{\text {new }}(\hat{x})^{T} r_{\text {new }}(\hat{x}) .
\end{aligned}
$$

Comparing the right-hand side of (75) with (53a), we recognize the product $J\left(\hat{x}_{\text {old }}\right)^{T} r_{\text {old }}\left(\hat{x}_{\text {old }}\right)$ as nothing more than $g=g_{\text {old }}$, the gradient direction vector of the original (i.e. unaugmented) system at the linearization point $\hat{x}_{\text {old }}$. Thus, (75) can be reduced to

$$
\bar{g}=\left(\begin{array}{l}
g \\
0
\end{array}\right)+J_{\text {new }}(\hat{x})^{T} r_{\text {new }}(\hat{x}) .
$$

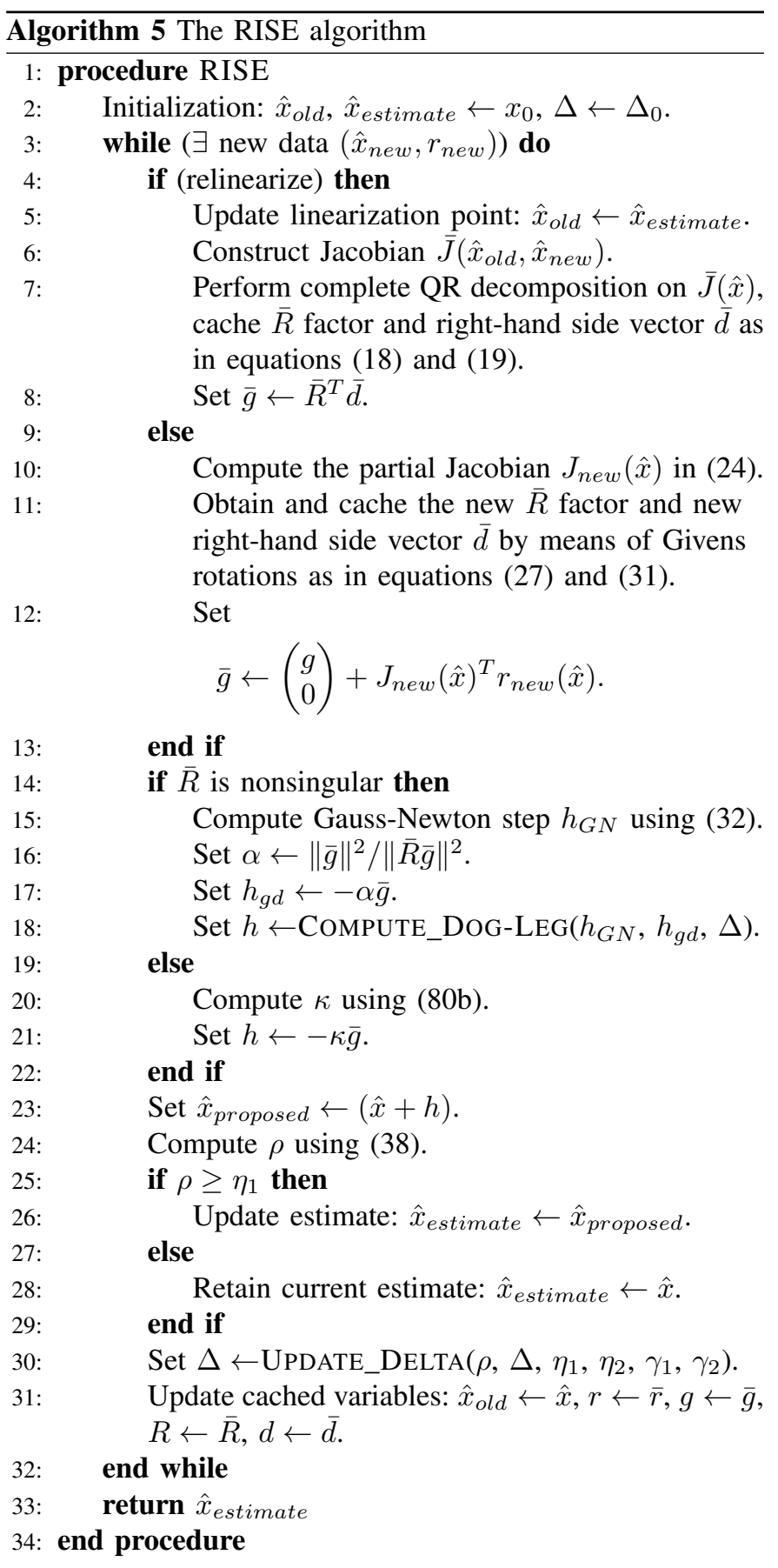

Since the matrix $J_{\text {new }}(\hat{x})$ is sparse and its row dimension is equal to the (small) number of new measurements added when the system is extended, equation (76) provides an efficient method for obtaining the gradient direction vector $\bar{g}$ for the augmented system by incrementally updating the previous gradient direction vector $g$, as desired.

Furthermore, in addition to obtaining $\bar{g}$ from $g$ using (76) in incremental update steps, we can also exploit computations already performed by iSAM to more efficiently batch-compute $\bar{g}$ during relinearization steps, when incremental updates cannot be performed. Substituting (29) into (53a), we obtain:

$$
\bar{g}=\left(\bar{Q}\left(\begin{array}{c}
\bar{R} \\
0
\end{array}\right)\right)^{T} \bar{r}(\hat{x})=\left(\begin{array}{ll}
\bar{R}^{T} & 0
\end{array}\right) \bar{Q}^{T} \bar{r}(\hat{x}) .
$$


Comparing (77) with (30) then shows that

$$
\bar{g}=\left(\begin{array}{ll}
\bar{R}^{T} & 0
\end{array}\right)\left(\begin{array}{l}
\bar{d} \\
\bar{e}
\end{array}\right)=\bar{R}^{T} \bar{d} .
$$

The advantage of equation (78) versus equation (53a) is that $\bar{R}$ is a sparse matrix of smaller dimension than $\bar{J}(\hat{x})$, so that the matrix-vector multiplication in (78) will be faster. Moreover, since iSAM already computes the factor $\bar{R}$ and the right-hand side vector $\bar{d}$, the factors on the right-hand side of (78) are available at no additional computational expense.

Having shown how to compute the vector $\bar{g}$, it remains only to determine the scaling factors $\alpha$ and $\kappa$ as in (53b) and (53c). The magnitude of $\bar{g}$ can of course be computed efficiently directly from $\bar{g}$ itself, which leaves only the denominator $\|\bar{J}(\hat{x}) \bar{g}\|^{2}$. To compute this quantity we again exploit the fact that iSAM already maintains the $\bar{R}$ factor of the $\mathrm{QR}$ decomposition for $\bar{J}(\hat{x})$; for since $\bar{Q}$ is orthogonal, then

$$
\|\bar{J}(\hat{x}) \bar{g}\|=\left\|\left(\bar{Q}\left(\begin{array}{c}
\bar{R} \\
0
\end{array}\right)\right) \bar{g}\right\|=\left\|\left(\begin{array}{c}
\bar{R} \\
0
\end{array}\right) \bar{g}\right\|=\|\bar{R} \bar{g}\|,
$$

and equations (53b) and (53c) are therefore equivalent to

$$
\begin{array}{ll}
\alpha & =\frac{\|\bar{g}\|^{2}}{\|\bar{R} \bar{g}\|^{2}}, \\
\kappa & = \begin{cases}\min \left\{\frac{\Delta}{\|\bar{g}\|}, \frac{\|\bar{g}\|^{2}}{\|\bar{R} \bar{g}\|^{2}}\right\}, & \|\bar{R} \bar{g}\|>0, \\
\frac{\Delta}{\|\bar{g}\|}, & \|\bar{R} \bar{g}\|=0 .\end{cases}
\end{array}
$$

Again, since $\bar{R}$ is sparse, the matrix-vector multiplication appearing in (80) is efficient.

Equations (76), (78), and (80) enable the implementation of RISE, a fully incrementalized version of the IGN-PDL algorithm that integrates directly into the existing iSAM framework (Algorithm 5).

\section{B. RISE2: Enabling fluid relinearization}

In this section we present RISE2, an improved version of RISE obtained by replacing iSAM with iSAM2 [10] in the derivation given in Section V-A. iSAM2 improves upon iSAM by eliminating the need to periodically reevaluate and refactor the entire Jacobian (two very expensive batch operations) in order to relinearize the system about its corrected estimate (cf. Section III-C); instead, iSAM2 efficiently relinearizes the system at every iteration by applying direct updates only to those (few) rows of $R$ and $d$ that are modified when relinearization occurs, a process known as fluid relinearization. Similarly, RISE2 eliminates expensive periodic batch factorizations (cf. lines 4 to 8 in Algorithm 5) by applying iSAM2's fluid relinearization in order to efficiently update $R, d$, and the gradient direction vector $g$ to their values at the updated estimate $\hat{x}_{\text {estimate }}$ at every iteration.

Internally, iSAM2 achieves efficient computation by means of the Bayes Tree [25], which encodes a symbolic factorization of the $R$ factor and right-hand side vector $d$ of the linear system (20) obtained from the QR decomposition (18), (19). Each node in the tree stores pairs of the form $\left[R_{i}, d_{i}\right]$, where

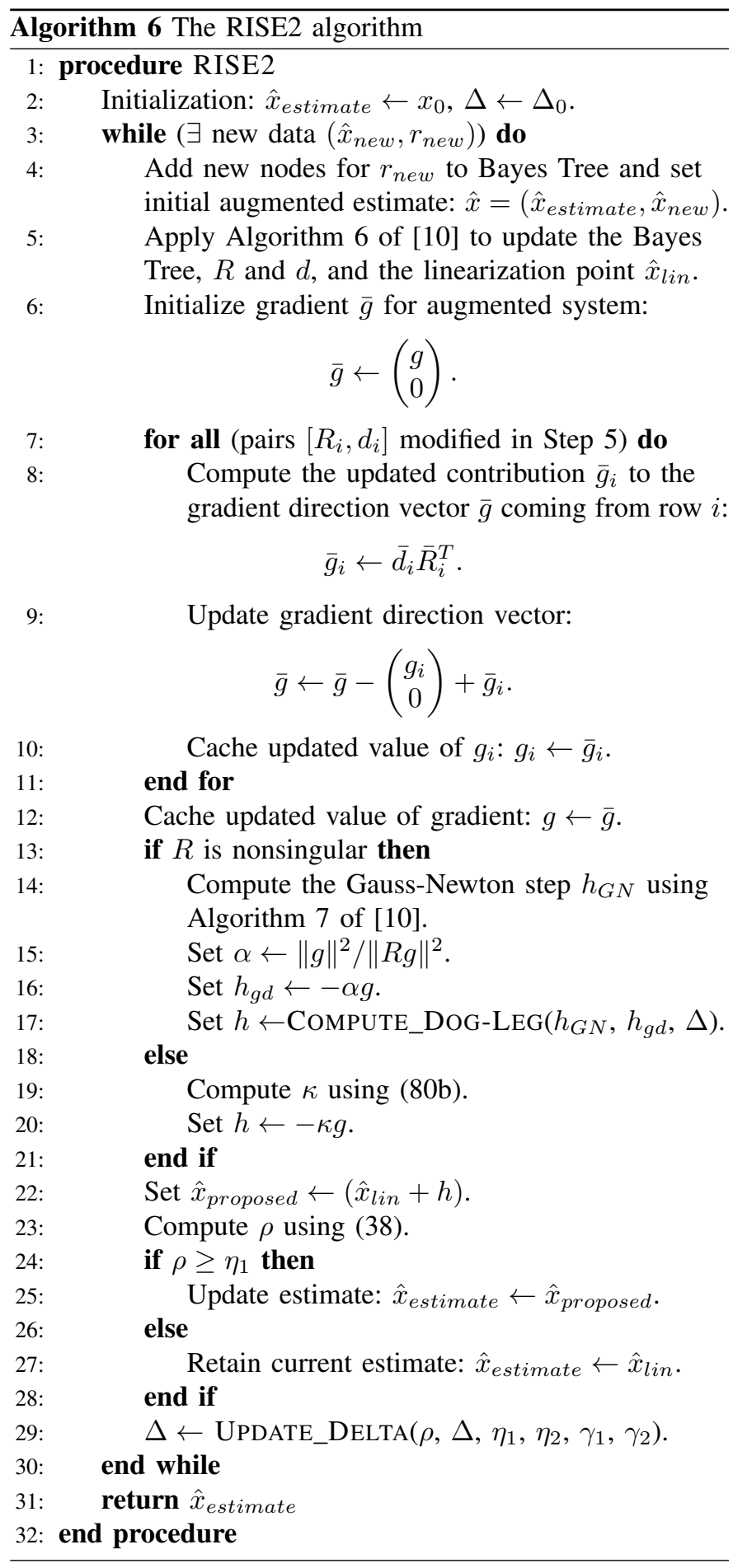

$R_{i} \in \mathbb{R}^{1 \times n}$ is the $i$ th row of the matrix $R$ and $d_{i} \in \mathbb{R}$ is the $i$ th element of the right-hand side vector $d$ :

$$
R=\left(\begin{array}{c}
R_{1} \\
\vdots \\
R_{n}
\end{array}\right) \in \mathbb{R}^{n \times n}, \quad d=\left(\begin{array}{c}
d_{1} \\
\vdots \\
d_{n}
\end{array}\right) \in \mathbb{R}^{n \times 1}
$$

(cf. Fig. 3(c) in [10]). Updating $R$ and $d$ by adding new measurements to the system or relinearizing the system about its updated estimate $\hat{x}_{\text {estimate }}$ can be implemented as simple, computationally efficient editing operations on the tree itself (cf. Algorithms 4 and 5 of [10], respectively); similarly, solv- 
ing the updated system for the Gauss-Newton step is achieved by means of a simple single-pass algorithm flowing from the root towards the leaves (Algorithm 7 of [10]). As in the case of the original RISE algorithm, we obtain RISE2 by exploiting these computations to produce an efficient incremental update rule for the gradient direction vector $g$.

According to (78), $g$ can be computed from $R$ and $d$ as:

$$
g=R^{T} d
$$

Substituting the block decomposition (81) into (82) produces

$$
g=R^{T} d=\left(\begin{array}{lll}
R_{1}^{T} & \cdots & R_{n}^{T}
\end{array}\right)\left(\begin{array}{c}
d_{1} \\
\vdots \\
d_{n}
\end{array}\right)=\sum_{i=1}^{n} d_{i} R_{i}^{T},
$$

which expresses $g$ as a linear combination of the rows of $R$.

When fluid relinearization and updating are applied, some of the values $\left[R_{i}, d_{i}\right]$ in the nodes of the Bayes Tree may be modified. By means of (83), we can recover the updated value $\bar{g}$ of the gradient direction vector corresponding to the new linearization point $\hat{x}_{\text {estimate }}$ using only those quantities that the Bayes Tree already computes during this update. Specifically, we initialize $\bar{g}$ according to:

$$
\bar{g} \leftarrow\left(\begin{array}{l}
g \\
0
\end{array}\right),
$$

and for each pair $\left[R_{i}, d_{i}\right]$ that was modified during the update of the Bayes Tree, we likewise update $\bar{g}$ according to:

$$
\bar{g} \leftarrow \bar{g}-\left(\begin{array}{c}
g_{i} \\
0
\end{array}\right)+\bar{g}_{i}
$$

where

$$
g_{i}=d_{i} R_{i}^{T}, \quad \bar{g}_{i}=\bar{d}_{i} \bar{R}_{i}^{T}
$$

here $\left[R_{i}, d_{i}\right]$ gives the values in row $i$ prior to their update, and $\left[\bar{R}_{i}, \bar{d}_{i}\right]$ gives these values after their update. Equations (85) and (86) indicate that the update to $\bar{g}$ due to row $i$ 's update simply consists of subtracting off the contribution $g_{i}$ to the gradient direction vector coming from row $i$ prior to its update and replacing it with the updated contribution $\bar{g}_{i}$.

Replacing the computation of $g$ in the original RISE algorithm (lines 4 to 13 of Algorithm 5) with the new incremental update procedure (84)-(86) produces RISE2 (Algorithm 6).

We point out that in both cases RISE(2)'s efficiency and incrementality are a direct result of exploiting iSAM(2)'s preexisting functionality for incrementally updating $R$ and $d$. In addition to being a purely intellectually pleasing result, this also means that any other computations depending upon preexisting iSAM(2) functionality (for example, online covariance extraction for data association [26]) can proceed with RISE(2) without modification.

\section{RELATED WORK}

There is a vast body of prior work on least-squares problems (1) and their solution; indeed, entire books have been devoted to this subject alone [27]. However, since our own interest in this problem is motivated by visual mapping applications, we will restrict our attention to prior work in the SLAM and bundle adjustment literature that attempts to solve (1) exactly.
In the SLAM literature, the first formulation of the full or smoothing problem as an instance of (1) is due to $\mathrm{Lu} \&$ Milios [28], who proposed to solve it using the Gauss-Newton algorithm. This approach remains perhaps the most popular, with many well-known algorithms [10], [29]-[33] differing only in how they solve the linear system (10) corresponding to (13). Lu \& Milios themselves originally proposed to solve (10) directly using dense matrix inversion, but the $O\left(n^{3}\right)$ computational cost of this technique is only tractable for fairly small problems. Subsequent work achieved improved computational speeds by exploiting symmetric positive definiteness and sparsity in the approximate Hessian (13b) using iterative methods such as sparse preconditioned conjugate gradient [29] or (multiresolution) Gauss-Seidel relaxation [30]. Thrun \& Montemerlo [31] exploited the sparse block structure of the Hessian in a direct method by using the Schur complement to solve first the (dense but low-dimensional) system corresponding to the robot pose update and then the (large but sparse) system corresponding to the landmark update. Grisetti et al. [32] exploited sparsity by implementing a hierarchical version of Gauss-Newton on a multiresolution pyramid and using lazy evaluation when propagating updates downward.

To further exploit sparsity, Dellaert \& Kaess [34] conducted a thorough investigation of direct linear-algebraic techniques for efficiently solving sparse linear systems of the form (10). One surprising result of this analysis was the primacy of variable ordering strategies as a factor in the computational cost of solving these systems; indeed, the use of fast sparse matrix factorization algorithms (such as sparse multifrontal QR [35] or Cholesky [36] decompositions) coupled with good variable ordering heuristics [21] enables the linear system (10) to be solved in a fraction of the time needed to simply construct it (i.e. evaluate the Jacobian or Hessian). This insight in turn eventually led to the development of the Bayes Tree [25] and iSAM2 [10], which directly update the reduced linear system (20) at each iteration rather than reevaluating and refactoring the Hessian in (10); this completely incremental approach is the current state of the art amongst Gauss-Newtonbased optimization algorithms in robotics.

Alternatively, some SLAM approaches propose to solve (1) using first-order methods. These methods have excellent robustness properties, but are limited to a linear convergence rate since they do not take advantage of curvature information. Olson et al. [37] proposed to overcome this slow convergence by using a deterministic variation of Jacobi-preconditioned stochastic gradient descent with a clever parameterization that enables the algorithm to make more rapid progress through the state space. While originally conceived as a batch algorithm, Olson's method was later adapted to the online setting through the use of spatially-varying learning rates and lazy evaluation [38]. Grisetti et al. [39] improved upon Olson's original parameterization by ordering variables using a spanning tree through the network of constraints rather than temporally. These approaches were later unified to produce TORO [40].

In bundle adjustment, the optimization (1) is typically solved in the batch (offline) case, and therefore BA solutions generally emphasize good robustness and convergence properties rather than real-time computational speed. To that end, 
many popular software packages used for BA (e.g. $\mathrm{g}^{2}$ o [33], SBA [41] and sSBA [42]) implement sparse batch versions of Levenberg-Marquardt to take advantage of its desirable robustness and numerical properties. One notable online approach is the continuable LM method of [43], which (like iSAM and RISE) applies a single update step per iteration and (also like RISE) maintains the value of the damping parameter $\lambda$ across iterations; however, unlike RISE (which incrementally updates the linear system (10)) it completely reconstructs and refactors the modified normal equations (74) in each iteration, which are the most expensive parts of computing each update step.

In summary, while prior work has addressed subsets of the following criteria, we believe that the RISE algorithm is the first to satisfy all three simultaneously:

- Speed: We implement a numerical optimization method with a superlinear convergence rate and incrementally update the linear system (32) across iterations rather than recomputing it, thus achieving computational speeds comparable to state-of-the-art online incremental sparse least-squares solvers.

- Robustness: Unlike purely Gauss-Newton-based methods, RISE is provably robust to highly nonlinear systems and numerically ill-conditioned Jacobians and is globally convergent for a broad class of objective functions.

- Generality: We place no restrictions on the admissible parameterizations of the state variables, nor on the number of arguments to each of the functions $r_{i}$.

\section{EXPERIMENTAL RESULTS}

In this section we illustrate the performance of the Powell's Dog-Leg, Gauss-Newton, and Levenberg-Marquardt batch methods and the $\operatorname{iSAM}(2)$ and RISE(2) incremental methods on a few representative optimization problems arising in the context of robotic mapping. We use the implementations of these algorithms (including preliminary research implementations of RISE and RISE2) available in the iSAM $^{1}$ and GTSAM $^{2}$ software libraries. Within each experiment, all algorithms are implemented atop a common set of data structures, so any differences in their performance are due solely to differences amongst the algorithms themselves.

Since our aim in these experiments is to characterize the performance of optimization algorithms, our primary experimental metrics will be the objective function value(s) obtained by each method and their total elapsed computation times. To that end, we have selected test problem instances for which the objective function is correctly specified (i.e. for which the data association is known and correct, either by construction in simulation or through the use of visual fiducials in realworld experiments) in order to experimentally control for the effects of front-end feature extraction and data association.

Finally, one of our principal motivations in developing an incremental optimization method that is more resilient to nonlinearity is to enable the use of robust cost functions in SLAM

\footnotetext{
${ }^{1}$ The iSAM Library (version 1.6), available through http://people.csail.mit. edu/kaess/isam/isam_v1_6.tgz.

${ }^{2}$ The GTSAM Library (version 2.1.0), available through https://research.cc. gatech.edu/borg/sites/edu.borg/files/downloads/gtsam-2.1.0.tgz
}

and visual mapping applications in order to attenuate the ill effects of occasional gross measurement errors. To that end, in the sequel we formulate M-estimation using a robust cost function $C(\delta) \geq 0$ as an instance of least-squares minimization by defining, for each raw residual $r_{i}(x)$, the corresponding robust least-squares residual $r_{i}^{\prime}(x)= \pm \sqrt{C\left(r_{i}(x)\right)}$; the M-estimate is then obtained as the minimizer of the least-squares problem (1) in the residuals $r_{i}^{\prime}(x)$.

\section{A. Simulated data: sphere 2500}

Here we consider the performance of the Powell's Dog-Leg, Gauss-Newton, and Levenberg-Marquardt batch methods and the iSAM and RISE incremental methods on 1000 randomlygenerated instances ${ }^{3}$ of the sphere 2500 dataset [10], a standard 6DOF pose-graph SLAM benchmark. In these experiments we use the implementations of these algorithms available in the iSAM v1.6 library with their default settings, and apply the pseudo-Huber robust cost function (cf. [2, Sec. A6.8]) with parameter $b=.5$.

1) Batch methods: In this experiment we compare the performance of the three batch methods to validate our adoption of Powell's Dog-Leg in Section IV as the sparse least-squares optimization method of choice. All algorithms use the same stopping criteria, and (in the interest of time) are limited to a maximum of 500 iterations. The initial estimate of the robot path is obtained by integrating the simulated raw odometry measurements. Results from the experiment are summarized in Table I, and the solutions computed by each algorithm for a single representative problem instance are shown in Fig. 5.

As expected, Powell's Dog-Leg and Levenberg-Marquardt obtain solutions of comparable quality, significantly outperforming Gauss-Newton. The superior performance of these algorithms can be understood in terms of their robust convergence properties and high tolerance for nonlinearity; it is clear from Table I that Gauss-Newton makes only marginal progress towards the minima in these examples.

Furthermore, in addition to its favorable accuracy, Powell's Dog-Leg is also the fastest algorithm of the three by an order of magnitude, both in terms of the number of iterations necessary to converge to a local minimum and the total elapsed computation time. In this case the superior speed of Powell's Dog-Leg versus Gauss-Newton is a consequence of its improved convergence; its superior speed versus LevenbergMarquardt has been studied previously [16], and is due in part to the fact that Powell's Dog-Leg need only solve (10) for the Newton step once at each linearization point, whereas Levenberg-Marquardt must solve the modified normal equations (74) (an expensive operation) whenever the linearization point is updated or the damping parameter $\lambda$ is changed.

2) Incremental methods: Next we compare the original iSAM algorithm with RISE (Algorithm 5); results are summarized in Table II (note that the statistics given for each method in the first and second rows of Table II are computed using only the set of problem instances for which that method ran to completion, as explained below).

\footnotetext{
${ }^{3}$ Generated using the generateSpheres ICRA2012. cpp executable in the iSAM v1.6 library.
} 


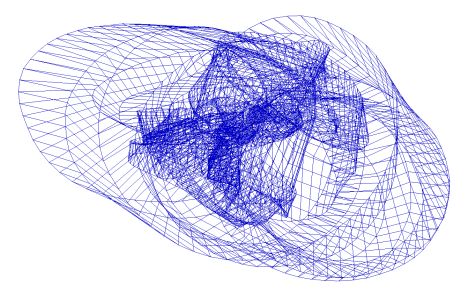

(a) Initial estimate

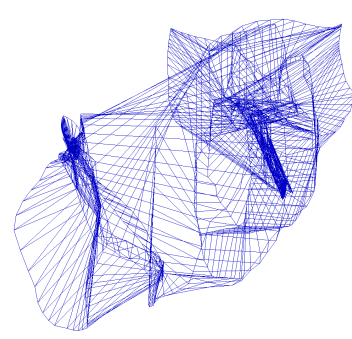

(b) Gauss-Newton

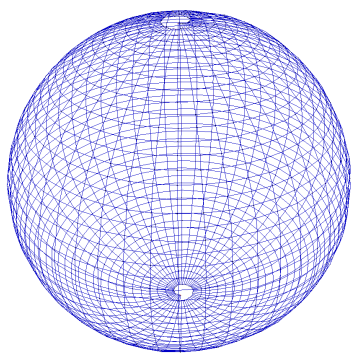

(c) Levenberg-Marquardt

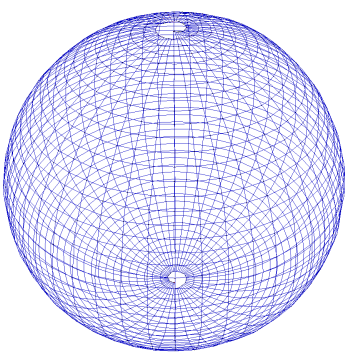

(d) Powell's Dog-Leg

Fig. 5. A representative instance of the sphere2500 6DOF SLAM problem from the batch experiments. 5(a): The initial estimate for the solution (objective function value 1.221 E8). 5(b): The solution obtained by Gauss-Newton (3.494 E6). 5(c): The solution obtained by Levenberg-Marquardt (8.306 E3). 5(d): The solution obtained by Powell's Dog-Leg (8.309 E3). Note that the objective function value for each of these solutions is within $\pm 0.5 \%$ of the median value for the corresponding method given in Table I.

\begin{tabular}{|c|c|c|c|c|c|c|c|c|c|}
\hline & \multicolumn{3}{|c|}{ Powell's Dog-Leg } & \multicolumn{3}{|c|}{ Gauss-Newton } & \multicolumn{3}{|c|}{ Levenberg-Marquardt } \\
\hline & Mean & Median & Std. Dev. & Mean & Median & Std. Dev. & Mean & Median & Std. Dev. \\
\hline Objective function value & $8.285 \mathrm{E} 3$ & $8.282 \mathrm{E} 3$ & 71.40 & $4.544 \mathrm{E} 6$ & $3.508 \mathrm{E} 6$ & $4.443 \mathrm{E} 6$ & $9.383 \mathrm{E} 3$ & $8.326 \mathrm{E} 3$ & $2.650 \mathrm{E} 3$ \\
\hline Computation time (sec) & 16.06 & 15.73 & 1.960 & 226.2 & 226.0 & 2.028 & 126.7 & 127.0 & 43.51 \\
\hline \# Iterations & 34.48 & 34 & 4.171 & 499.9 & 500 & 2.500 & 338.2 & 328 & 138.9 \\
\hline \# Iteration limit reached & \multicolumn{3}{|c|}{0} & \multicolumn{3}{|c|}{998} & \multicolumn{3}{|c|}{311} \\
\hline
\end{tabular}

TABLE I

SUMMARY OF RESULTS FOR SPHERE2500 BATCH EXPERIMENTS

\begin{tabular}{|c|c|c|c|c|c|c|}
\hline & \multicolumn{3}{|c|}{ RISE } & \multicolumn{3}{|c|}{ iSAM } \\
\hline & Mean & Median & Std. Dev. & Mean & Median & Std. Dev. \\
\hline Objective function value & $9.292 \mathrm{E} 3$ & $9.180 \mathrm{E} 3$ & $5.840 \mathrm{E} 2$ & $6.904 \mathrm{E} 11$ & $1.811 \mathrm{E} 4$ & $1.242 \mathrm{E} 13$ \\
\hline Computation time (sec) & 50.21 & 50.18 & 0.13 & 42.97 & 42.95 & 0.13 \\
\hline \# Rank-deficient Jacobians & \multicolumn{3}{|c|}{$0(0.0 \%)$} & \multicolumn{3}{|c|}{$586(58.6 \%)$} \\
\hline
\end{tabular}

TABLE II

SUMMARY OF RESULTS FOR SPHERE2500 INCREMENTAL EXPERIMENTS

As expected, RISE significantly outperformed iSAM in terms of final solution quality. In over half of the problem instances, the solution computed by iSAM diverged so far from the true minimum that the numerically-computed Jacobian became rank-deficient, forcing the algorithm to abort the computation (solving equation (20) for the Gauss-Newton step requires that the Jacobian be full-rank). Even for those problem instances in which iSAM ran to completion (which are necessarily the instances that are the "easiest" to solve), Table II shows that the solutions computed using the incremental Gauss-Newton approach have significantly greater costs than those computed using the incremental Powell's Dog-Leg method. Indeed, RISE's performance on all of the problem instances was, on the average, significantly better than iSAM's performance on only the easiest instances.

RISE's enhanced robustness versus iSAM does come at a slightly greater computational cost: each iteration of the RISE algorithm must compute the Gauss-Newton step (the output of iSAM) as an intermediate result in the computation of the dog-leg step. As shown in Algorithms 3 and 5, the cost of computing the dog-leg step given the Gauss-Newton step is dominated by the costs of the matrix-vector multiplications needed to compute the gradient descent step; since these have the same asymptotic time-complexity as the backsubstitution that iSAM already performs to compute the Gauss-Newton step, we expect that RISE will suffer at most a small constantfactor slowdown in speed versus iSAM. The results in Table II show that in practice this constant-factor slowdown has only a modest effect on RISE's overall execution speed (an increase of about $20 \%$ versus iSAM) when the computational costs of manipulating the underlying data structures are also included: both iSAM and RISE are fast enough to run comfortably in real-time.

\section{B. Visual mapping with a calibrated monocular camera}

In this experiment we consider a significantly more challenging test scenario: visual mapping with a calibrated monocular camera via incremental bundle adjustment. Bundle adjustment is known to suffer from a litany of numerical challenges, including strong nonlinearities in the objective function (due to the nonlinear camera projection mappings, the rotational degrees of freedom in the camera pose estimates, and the use of robust cost functions) and poor numerical conditioning (which can be caused by unfavorable camera configurations or large variations across the uncertainties of the reconstructed camera poses and point positions) [3]. Successful bundle adjustment optimization methods must be able to robustly address these numerical difficulties.

The input for this experiment consists of a short (141 second) $640 \times 480$ monochrome video sequence recorded by the left camera of a Bumblebee2 stereocamera as it was hand-scanned over a room-sized static scene containing 46 AprilTags [44]. A set of keyframes and point observations was extracted from this video by sampling at $1 \mathrm{~Hz}$ (to provide a reasonable baseline between successive keyframes) and then 


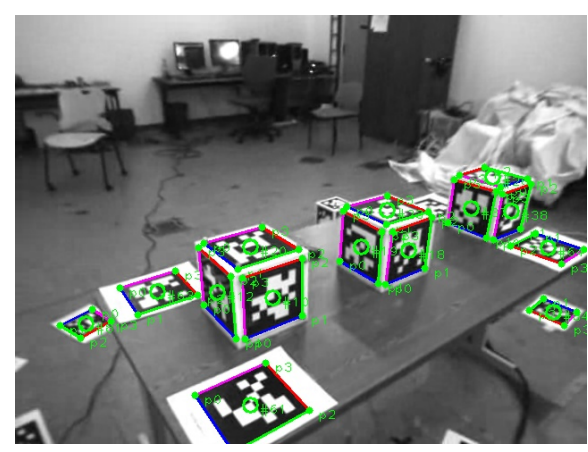

(a) Keyframe 96

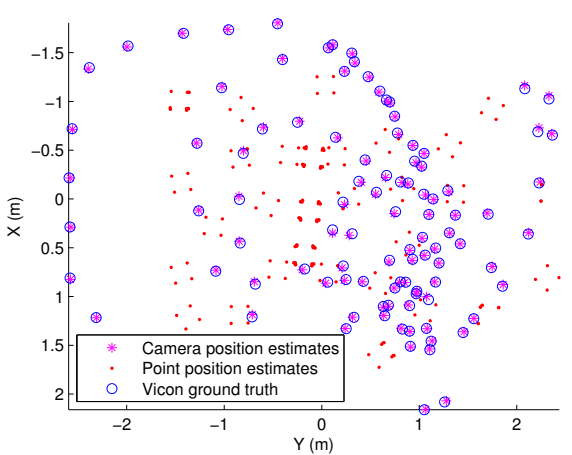

(b) Final RISE2 BA solution

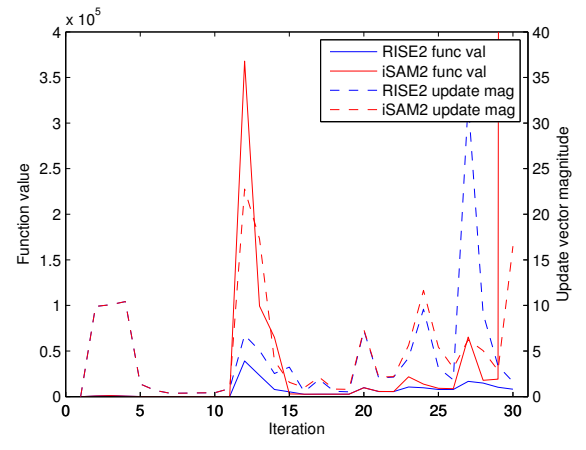

(c) Incremental optimization

Fig. 6. Visual mapping with a calibrated monocular camera. (a): A graphical overlay highlighting 14 detected AprilTags and the estimated positions of their corner points in keyframe 96, one of 104 keyframes extracted from the input video. (b): An overhead view of the final camera and point position estimates obtained via incremental bundle adjustment using RISE2, registered against the Vicon system ground truth. (c): A plot of the objective function values and update vector magnitudes computed by iSAM2 and RISE2 for the first 30 iterations of the visual mapping task (the iSAM2 algorithm aborted the computation in iteration 31 with a numerically rank-deficient Jacobian).

\begin{tabular}{|c|c|c|c|c|c|c|c|c|c|}
\hline & \multicolumn{3}{|c|}{ RISE2 (final) } & \multicolumn{3}{|c|}{ RISE2 (iteration 30) } & \multicolumn{3}{|c|}{ iSAM2 (iteration 30) } \\
\hline & Mean & Median & Std. Dev. & Mean & Median & Std. Dev. & Mean & Median & Std. Dev. \\
\hline Objective function value & \multicolumn{3}{|c|}{$5.588 \mathrm{E} 2$} & \multicolumn{3}{|c|}{$8.157 \mathrm{E} 3$} & \multicolumn{3}{|c|}{$1.574 \mathrm{E} 7$} \\
\hline Computation time (sec) & \multicolumn{3}{|c|}{$1.040 \mathrm{E} 2$} & \multicolumn{3}{|c|}{$8.618 \mathrm{E}-1$} & \multicolumn{3}{|c|}{$7.746 \mathrm{E}-1$} \\
\hline Camera position errors (m) & $1.574 \mathrm{E}-2$ & $1.384 \mathrm{E}-2$ & $8.209 \mathrm{E}-3$ & $1.844 \mathrm{E}-1$ & $6.170 \mathrm{E}-2$ & $5.855 \mathrm{E}-1$ & $3.164 \mathrm{E}-1$ & $4.117 \mathrm{E}-2$ & $6.967 \mathrm{E}-1$ \\
\hline
\end{tabular}

TABLE III

SUMMARY OF RESULTS FOR INCREMENTAL BUNDLE ADJUSTMENT

selecting as keyframes all images in which at least 3 AprilTags were detected; this yielded 104 keyframes containing 3876 observations of the 184 AprilTag corner points. These observations were used to estimate the 3D camera pose of each keyframe and the 3D position of each corner point via bundle adjustment (i.e. by minimizing the sum of reprojection errors under the Huber robust cost function (cf. [2, Sec. A6.8]) with parameter $b=1$ ); the camera's internal calibration was estimated using Zhang's method [45] immediately prior to recording the video and treated as a fixed constant. No prior information about the AprilTags' geometry was included in the adjustment; the tags were used only to solve the data association problem, which is beyond the scope of this work. Ground truth was also acquired by tracking the position of the camera using a Vicon system. ${ }^{4}$

Incremental bundle adjustment was performed using the iSAM2 and RISE2 implementations available in the GTSAM v2.1.0 library (here one iteration comprised the incorporation of all observations from a single keyframe). Camera poses were initialized using the EPnP algorithm [46] (whenever at least four previously-initialized points were in view) or the two-view homogeneous DLT method of [2, Sec. 9.6]; points were initialized using either the homogeneous DLT triangulation method of [2, Sec. 12.2] or the two-view homogeneous method of [2, Sec. 9.6]. Results from the experiment are shown in Fig. 6 and Table III.

The RISE2 algorithm successfully processed all 104 frames, converging to a final solution with an objective function value of 558.8 and a raw RMS reprojection error of .3797 pixels. In contrast, the iSAM2 algorithm did not even run to completion

\footnotetext{
${ }^{4}$ The complete dataset for this experiment (including ground truth) is available through http://groups.csail.mit.edu/marine/apriltags_groundtruth_BA/
}

on this example; in the 31st iteration it reported that the Jacobian $J\left(x^{(31)}\right)$ was numerically rank-deficient and aborted the computation. A closer investigation reveals that the GaussNewton update step applied by iSAM2 in iteration 30 overshot the true objective function minimum by moving 5 points behind several of the cameras from which they were visible near the newly-initialized camera for keyframe 30 (cf. Fig. 6(c) and Table III); this led to a poor initialization of the camera pose for keyframe 31 and the subsequent failure of the algorithm.

Our experience has been that this failure mode (overshooting followed by the rank-deficiency of the Jacobian computed in the next iteration) is generic when $\operatorname{iSAM}(2)$ is applied to highly nonlinear or ill-conditioned systems. In contrast, RISE(2)'s use of the trust-region approach enables it to reliably overcome these numerical challenges.

\section{CONCLUSION}

In this paper we presented RISE, an incremental trust-region method for robust online sparse least-squares estimation. RISE improves upon current state-of-the-art sequential sparse leastsquares solvers by providing superior robustness to objective function nonlinearity and numerical ill-conditioning while simultaneously exploiting prior advances in incremental optimization to achieve fast online computation. In addition to deriving the RISE algorithm itself, we also provided a thorough theoretical analysis of its desirable numerical properties and proved its global convergence for a broad class of inferential cost functions (twice-continuously differentiable functions with bounded sublevel sets). Finally, we evaluated the algorithm empirically in simulation using standard 6DOF pose-graph SLAM benchmark datasets, and demonstrated its 
superior performance on a more challenging real-world example arising in the context of online visual mapping.

In addition to its utility in SLAM and visual mapping tasks, we believe that the RISE algorithm can also be advantageously applied to other numerically challenging online inference problems sharing the common mathematical structure (2); we are particularly interested in its use as a superlinear optimization method for online machine learning with kernels [5]. Recent work in the robotics [47] and computer vision [48] literature has shown promising results in this direction, but utilizes Gauss-Newton-based approaches that (as demonstrated herein) limit the class of objective functions that can be reliably employed. It would be interesting to consider applications of RISE (and related techniques) in the context of online machine learning in future work.

\section{APPENDIX}

Proof of Theorem 3: We prove Theorem 3 by means of Theorem 2. Since $\mathcal{L}_{S}\left(x^{(0)}\right)$ is bounded, there exists $D>0$ such that $\left\|x-x^{(0)}\right\|<D$ for all $x \in \mathcal{L}_{S}\left(x^{(0)}\right)$. Define the convex open set

$$
\Omega=\left\{x \in \mathbb{R}^{n} \mid\left\|x-x^{(0)}\right\|<D\right\} .
$$

Then $\bar{\Omega}$ is a closed and bounded (hence compact) subset of $\mathbb{R}^{n}$ containing the sublevel set $\mathcal{L}_{S}\left(x^{(0)}\right)$. Equation (11b) gives the elements of the Hessian matrix $\frac{\partial^{2} S}{\partial x^{2}}$, which are continuous since $r \in C^{2}$, and therefore $\left\|\frac{\partial^{2} S}{\partial x^{2}}\right\|$ attains a maximum value $L$ on the compact set $\bar{\Omega} ; L$ is thus a Lipschitz constant for $\nabla S$ on $\Omega$. The same argument applied to the approximate Hessians $B$ whose elements are defined by equation (12) shows that $\|B\|$ likewise attains a maximum $\beta$ on $\bar{\Omega}$. Finally, $S$ (as a sum-ofsquares function) is clearly lower-bounded by 0 everywhere. This establishes the initial hypotheses of Theorem 2.

We now check each of the enumerated criteria (i)-(v) in turn. Condition (i) is immediate from the definitions of the dog-leg and constrained Cauchy steps in and (46) and (50). Condition (ii) is satisfied with $\zeta=0$ since the gradients used in Algorithm 4 are exact (cf. equation (13a)). Condition (iii) is satisfied using the value of $\beta$ obtained in the preceding paragraph. Condition (iv) is trivially satisfied in the case of the Cauchy step $h_{C}$, since this step is by definition always antiparallel to the gradient (cf. equation (50)). In the case of the dog-leg step, we observe using (44) that

$$
\left\|h_{g d}\right\|=\left\|-\frac{\|g\|^{2}}{g^{T} B g} g\right\|=\frac{\|g\|^{3}}{g^{T} B g} \geq \frac{\|g\|^{3}}{\beta\|g\|^{2}}=\frac{\|g\|}{\beta},
$$

so that (by virtue of the computation in Algorithm 3) $h_{d l}$ is also antiparallel to the gradient whenever

$$
\Delta^{(i)}<\frac{\left\|g^{(i)}\right\|}{\beta} \leq\left\|h_{g d}^{(i)}\right\| .
$$

Equation (89) will hold for all $i>N$ for some sufficiently large $N$ by virtue of the additional hypotheses (56) in effect for the purposes of this condition.

Finally, for condition (v), consider the predicted decrease for the Cauchy step $h_{C}=-\kappa g$ defined in (50) and (51):

$$
\operatorname{pred}(h)=\kappa\|g\|^{2}-\frac{1}{2} \kappa^{2} g^{T} B g \text {. }
$$

The full (i.e. unconstrained) Cauchy step has $\kappa=\frac{\|g\|^{2}}{g^{T} B g}$, in which case (90) becomes:

$$
\begin{aligned}
\operatorname{pred}(h) & =\left(\frac{\|g\|^{2}}{g^{T} B g}\right)\|g\|^{2}-\frac{1}{2}\left(\frac{\|g\|^{2}}{g^{T} B g}\right)^{2} g^{T} B g \\
& =\frac{1}{2} \cdot \frac{\|g\|^{4}}{g^{T} B g} \geq \frac{\|g\|^{2}}{2 \beta}
\end{aligned}
$$

since $\|B\| \leq \beta$. In the constrained case with $g^{T} B g>0$, $\kappa=\Delta /\|g\|$ only when $\Delta /\|g\|<\|g\|^{2} / g^{T} B g$ (implying $\Delta g^{T} B g<\|g\|^{3}$ ), in which case (90) simplifies as

$$
\begin{aligned}
\operatorname{pred}(h) & =\left(\frac{\Delta}{\|g\|}\right)\|g\|^{2}-\frac{1}{2}\left(\frac{\Delta}{\|g\|}\right)^{2} g^{T} B g \\
& =\Delta\|g\|-\frac{1}{2} \frac{\Delta}{\|g\|^{2}}\left(\Delta g^{T} B g\right) \\
& \geq \frac{1}{2} \Delta\|g\| .
\end{aligned}
$$

Finally, for the constrained case with $g^{T} B g \leq 0$, we observe that in fact $g^{T} B g=0$ since the Gauss-Newton Hessian approximation (13b) is always positive semidefinite; thus $\kappa=\frac{\Delta}{\|g\|}$ and (90) simplifies as

$$
\operatorname{pred}(h)=\left(\frac{\Delta}{\|g\|}\right)\|g\|^{2}=\Delta\|g\| .
$$

Equations (91)-(93) show that the Cauchy step defined in (50) satisfies the uniform predicted decrease condition (59) with $c=1$ and $\sigma=\beta$. For the dog-leg step, we simply observe that by virtue of (45), (46) and Lemma $1, \operatorname{pred}\left(h_{d l}\right) \geq \operatorname{pred}\left(h_{C}\right)$ whenever $h_{d l}$ exists. This proves condition (v), and completes the proof.

\section{ACKNOWLEDGMENTS}

The authors would like to thank F. Dellaert and R. Roberts for the RISE2 implementation in the GTSAM library.

\section{REFERENCES}

[1] S. Thrun, W. Burgard, and D. Fox, Probabilistic Robotics. Cambridge, MA: The MIT Press, 2008.

[2] R. Hartley and A. Zisserman, Multiple View Geometry in Computer Vision, 2nd ed. Cambridge, UK: Cambridge University Press, 2004.

[3] B. Triggs, P. McLauchlan, R. Hartley, and A. Fitzgibbon, "Bundle adjustment - a modern synthesis," in Vision Algorithms: Theory and Practice, ser. Lecture Notes in Computer Science, W. Triggs, A. Zisserman, and R. Szeliski, Eds., vol. 1883. Springer Verlag, 2000, pp. 298-372.

[4] C. Bishop, Pattern Recognition and Machine Learning. New York: Springer Science+Business Media, 2006

[5] B. Schölkopf and A. Smola, Learning with Kernels. Cambridge, MA: The MIT Press, 2002.

[6] K. Levenberg, "A method for the solution of certain nonlinear problems in least squares," Quart. Appl. Math, vol. 2, pp. 164-168, 1944.

[7] D. Marquardt, "An algorithm for least-squares estimation of nonlinear parameters," J. Soc. Indust. Appl. Math., vol. 11, no. 2, pp. 431-441, Jun. 1963

[8] J. Nocedal and S. Wright, Numerical Optimization, 2nd ed. New York: Springer Science+Business Media, 2006

[9] M. Kaess, A. Ranganathan, and F. Dellaert, "iSAM: Incremental smoothing and mapping," IEEE Trans. Robotics, vol. 24, no. 6, pp. 1365-1378, Dec. 2008.

[10] M. Kaess, H. Johannsson, R. Roberts, V. Ila, J. Leonard, and F. Dellaert, "iSAM2: Incremental smoothing and mapping using the Bayes tree," Intl. J. of Robotics Research, vol. 31, no. 2, pp. 216-235, Feb. 2012.

[11] R. Fletcher, Practical Methods of Optimization, 2nd ed. John Wiley \& Sons, 1987. 
[12] M. Powell, "A new algorithm for unconstrained optimization," in Nonlinear Programming, J. Rosen, O. Mangasarian, and K. Ritter, Eds. London: Academic Press, 1970, pp. 31-65.

[13] R. Carter, "On the global convergence of trust region algorithms using inexact gradient information," SIAM J. Numer. Anal., vol. 28, no. 1, pp. 251-265, Feb. 1991.

[14] M. Powell, "On the global convergence of trust region algorithms for unconstrained minimization," Math. Program., vol. 29, no. 3, pp. 297 303, 1984.

[15] G. Shultz, R. Schnabel, and R. Byrd, "A family of trust-region-based algorithms for unconstrained minimization with strong global convergence properties," SIAM J. Numer. Anal., vol. 22, no. 1, pp. 47-67, Feb. 1985.

[16] M. Lourakis and A. Argyros, "Is Levenberg-Marquardt the most efficient optimization algorithm for implementing bundle adjustment?" Intl. Conf. on Computer Vision (ICCV), vol. 2, pp. 1526-1531, 2005.

[17] F. Kschischang, B. Frey, and H.-A. Loeliger, "Factor graphs and the sum-product algorithm," IEEE Trans. Inf. Theory, vol. 47, no. 2, pp. 498-519, Feb. 2001.

[18] D. Rosen, M. Kaess, and J. Leonard, "Robust incremental online inference over sparse factor graphs: Beyond the Gaussian case," in IEEE Intl. Conf. on Robotics and Automation (ICRA), Karlsruhe, Germany, May 2013, pp. 1017-1024.

[19] G. Golub and C. V. Loan, Matrix Computations, 3rd ed. Baltimore, MD: Johns Hopkins University Press, 1996.

[20] S. Strogatz, Nonlinear Dynamics and Chaos. Cambridge, MA: Perseus Books Publishing, 2001.

[21] T. Davis, J. Gilbert, S. Larimore, and E. Ng, "A column approximate minimum degree ordering algorithm," ACM Trans. Math. Softw., vol. 30, no. 3, pp. 353-376, Sep. 2004

[22] J. Moré and D. Sorensen, "Computing a trust region step," SIAM J. Sci. Stat. Comput., vol. 4, no. 3, Sep. 1983.

[23] A. Tikhonov and V. Arsenin, Solutions of Ill-Posed Problems. Washington, D.C.: V.H. Winston \& Sons, 1977.

[24] D. Rosen, M. Kaess, and J. Leonard, "An incremental trust-region method for robust online sparse least-squares estimation," in IEEE Intl. Conf. on Robotics and Automation (ICRA), St. Paul, MN, May 2012, pp. 1262-1269.

[25] M. Kaess, V. Ila, R. Roberts, and F. Dellaert, "The Bayes tree: An algorithmic foundation for probabilistic robot mapping," in Intl. Workshop on the Algorithmic Foundations of Robotics, WAFR, Singapore, Dec. 2010.

[26] M. Kaess and F. Dellaert, "Covariance recovery from a square root information matrix for data association," Journal of Robotics and Autonomous Systems, vol. 57, no. 12, pp. 1198-1210, Dec. 2009.

[27] A. Björck, Numerical Methods for Least Squares Problems. Philadelphia: The Society for Industrial and Applied Mathematics (SIAM), 1996.

[28] F. Lu and E. Milios, "Globally consistent range scan alignment for environmental mapping," Autonomous Robots, vol. 4, pp. 333-349, Apr. 1997.

[29] K. Konolige, "Large-scale map-making," in Proceedings of the National Conference on Artificial Intelligence. AAAI Press / The MIT Press, 2004, pp. 457-463.

[30] U. Frese, P. Larsson, and T. Duckett, "A multilevel relaxation algorithm for simultaneous localization and mapping," IEEE Trans. Robotics, vol. 21, no. 2, Apr. 2005.

[31] S. Thrun and M. Montemerlo, "The GraphSLAM algorithm with applications to large-scale mapping of urban structures," Intl. J. of Robotics Research, vol. 25, no. 5-6, pp. 403-429, 2006.

[32] G. Grisetti, R. Kümmerle, C. Stachniss, U. Frese, and C. Hertzberg, "Hierarchical optimization on manifolds for online 2D and 3D mapping," in IEEE Intl. Conf. on Robotics and Automation (ICRA), Anchorage, AK, May 2010, pp. 273-278.

[33] R. Kümmerle, G. Grisetti, H. Strasdat, K. Konolige, and W. Burgard, "g2o: A general framework for graph optimization," in IEEE Intl. Conf. on Robotics and Automation (ICRA), Shanghai, China, May 2011, pp. 3607-3613.

[34] F. Dellaert and M. Kaess, "Square Root SAM: Simultaneous localization and mapping via square root information smoothing," Intl. J. of Robotics Research, vol. 25, no. 12, pp. 1181-1203, Dec. 2006.

[35] P. Matstoms, "Sparse QR factorization in MATLAB," ACM Trans. Math. Softw., vol. 20, no. 1, pp. 136-159, Mar. 1994.

[36] Y. Chen, T. Davis, W. Hager, and S. Rajamanickam, "Algorithm 887: CHOLMOD, supernodal sparse Cholesky factorization and update/downdate," ACM Trans. Math. Softw., vol. 35, no. 3, pp. 22:1-22:14, Oct. 2008.
[37] E. Olson, J. Leonard, and S. Teller, "Fast iterative alignment of pose graphs with poor initial estimates," in IEEE Intl. Conf. on Robotics and Automation (ICRA), Orlando, FL, May 2006, pp. 2262-2269.

[38] E. Olson, O. Brock, and C. Stachniss, "Spatially-adaptive learning rates for online incremental SLAM," in Robotics: Science and Systems (RSS), Jun. 2007.

[39] G. Grisetti, C. Stachniss, S. Grzonka, and W. Burgard, "A tree parameterization for efficiently computing maximum likelihood maps using gradient descent," in Robotics: Science and Systems (RSS), 2007.

[40] G. Grisetti, D. Rizzini, C. Stachniss, E. Olson, and W. Burgard, "Online constraint network optimization for efficient maximum likelihood map learning," in IEEE Intl. Conf. on Robotics and Automation (ICRA), Pasadena, CA, May 2008, pp. 1880-1885.

[41] M. Lourakis and A. Argyros, "SBA: A software package for generic sparse bundle adjustment," ACM Trans. Math. Softw., vol. 36, no. 1, pp. 1-30, Mar. 2009

[42] K. Konolige, "Sparse sparse bundle adjustment," in BMVC, 2010, pp. $1-11$.

[43] K. Konolige, G. Grisetti, R. Kümmerle, W. Burgard, B. Limketkai, and R. Vincent, "Efficient sparse pose adjustment for 2D mapping," in IEEE/RSJ Intl. Conf. on Intelligent Robots and Systems (IROS), Taipei, Taiwan, Oct. 2010, pp. 22-29.

[44] E. Olson, "AprilTag: A robust and flexible visual fiducial system," in IEEE Intl. Conf. on Robotics and Automation (ICRA), Shanghai, China, May 2011, pp. 3400-3407.

[45] Z. Zhang, "A flexible new technique for camera calibration," IEEE Trans. Pattern Analysis and Machine Intelligence, vol. 22, no. 11, pp. 13301334, Nov. 2000

[46] V. Lepetit, F. Moreno-Noguer, and P. Fua, "EPnP: An accurate $O(n)$ solution to the PnP problem," International Journal of Computer Vision, vol. 81, pp. 155-166, 2009.

[47] C. Tong, P. Furgale, and T. Barfoot, "Gaussian process Gauss-Newton for non-parametric simultaneous localization and mapping," Intl. J. of Robotics Research, vol. 32, no. 5, pp. 507-525, May 2013.

[48] A. Ranganathan, M.-H. Yang, and J. Ho, "Online sparse Gaussian process regression and its applications," IEEE Trans. on Image Processing, vol. 20, no. 2, pp. 391-404, Feb. 2011.

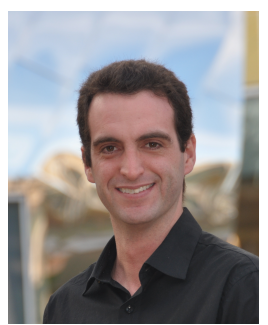

David M. Rosen is a doctoral candidate in Computer Science at MIT's Computer Science and Artificial Intelligence Laboratory. His research explores the mathematical foundations of robotic perception, including optimization, probabilistic inference, and machine learning. He holds the degrees of B.S. in Mathematics (with a minor in Control and Dynamical Systems) from the California Institute of Technology (2008) and M.A. in Mathematics from the University of Texas at Austin (2010).

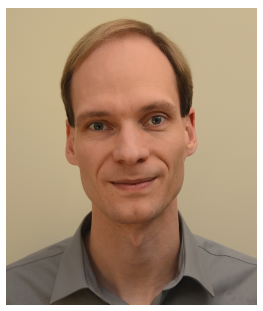

Michael Kaess is Assistant Research Professor in the Robotics Institute at Carnegie Mellon University. Previously he has been a research scientist and postdoctoral associate in the MIT Computer Science and Artificial Intelligence Laboratory (CSAIL). His research focuses on robot perception for navigation and autonomy. He received the Ph.D. (2008) and M.S. (2002) degrees in Computer Science from the Georgia Institute of Technology, Atlanta, GA.

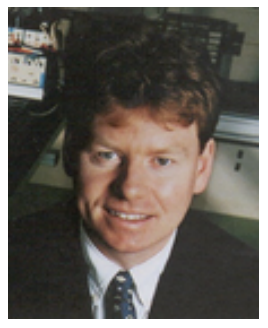

John J. Leonard is Professor of Mechanical and Ocean Engineering in the MIT Department of Mechanical Engineering and a member of the MIT Computer Science and Artificial Intelligence Laboratory (CSAIL). His research addresses the problems of navigation and mapping for autonomous mobile robots. He holds the degrees of B.S.E.E. in Electrical Engineering and Science from the University of Pennsylvania (1987) and D.Phil. in Engineering Science from the University of Oxford (formally 1994). He has served as an associate editor of the IEEE Journal of Oceanic Engineering and of the IEEE Transactions on Robotics and Automation. Currently, he serves as Area Head for Ocean Science and Engineering in the MIT Department of Mechanical Engineering and as Co-Director of the Ford-MIT Alliance. He is the recipient of an NSF Career Award (1998), an E.T.S. Walton Visitor Award from Science Foundation Ireland (2004), and the King-Sun Fu Memorial Best Transactions on Robotics Paper Award (2006). 